\title{
On the Strong Ellipticity of the Anisotropic Linearly Elastic Materials
}

\author{
Stan Chiriţă • Alexandre Danescu • Michele Ciarletta
}

Received: 10 July 2006 / Accepted: 28 November 2006 /

Published online: 12 January 2007

(C) Springer Science + Business Media B.V. 2007

\begin{abstract}
In this paper we derive necessary and sufficient conditions for strong ellipticity in several classes of anisotropic linearly elastic materials. Our results cover all classes in the rhombic system (nine elasticities), four classes of the tetragonal system (six elasticities) and all classes in the cubic system (three elasticities). As a special case we recover necessary and sufficient conditions for strong ellipticity in transversely isotropic materials. The central result shows that for the rhombic system strong ellipticity restricts some appropriate combinations of elasticities to take values inside a domain whose boundary is the third order algebraic surface defined by $x^{2}+y^{2}+z^{2}-2 x y z-1=0$ situated in the cube $|x|<1,|y|<1,|z|<1$. For more symmetric situations, the general analysis restricts combinations of elasticities to range inside either a plane domain (for four classes in the tetragonal system) or in an one-dimensional interval (for the hexagonal systems, transverse isotropy and cubic system). The proof involves only the basic statement of the strong ellipticity condition.
\end{abstract}

Key words strong ellipticity $\cdot$ anisotropic materials $\cdot$ rhombic $\cdot$ tetragonal $\cdot$ cubic . hexagonal systems $\cdot$ transverse isotropy

Mathematics Subject Classifications (2000) 74 B $05 \cdot 74$ E 10

S. Chiriţă $(\bowtie)$

Faculty of Mathematics, “Al. I. Cuza” University, Blvd. Carol I, no. 11, 700506-Iaşi, Romania e-mail: schirita@uaic.ro

A. Danescu

Department Mécanique des Solides, Génie Mécanique, Génie Civil,

Ecole Centrale de Lyon, Av. Guy de Collongue, BP 163-69131 Ecully Cedex, France

M. Ciarletta

Department of Engineering Information and Applied Mathematics, University of Salerno, 84084 Fisciano (SA), Italy 


\section{Introduction}

For a linearly anisotropic elastic solid the components $C_{i j k l}$ of the tensor of elastic moduli satisfy the symmetries

$$
C_{i j k l}=C_{k l i j}=C_{i j l k},
$$

while the indices $i, j, k, l$ take values $1,2,3$.

The strong ellipticity condition states that

$$
C_{i j k l} n_{i} n_{k} m_{j} m_{l}>0
$$

for all non-zero vectors $\mathbf{m}=\left(m_{1}, m_{2}, m_{3}\right)$ and $\mathbf{n}=\left(n_{1}, n_{2}, n_{3}\right)$, where summation is implied by index repetition. This condition is of importance in discussing uniqueness, wave propagation (see e.g. Gurtin [1, p. 86]), loss of ellipticity in the context of the nonlinear elasticity of fibre-reinforced materials (see Merodio and Ogden [2]) and also in the study of spatial behaviour of the constrained anisotropic cylinders (see Chiriţă and Ciarletta [3]).

In the particular case of an isotropic linearly elastic material with Lamé moduli $\lambda$ and $\mu$ the strong ellipticity is equivalent to the fact that (see e.g. Gurtin $[1$, p. 86])

$$
\mu>0, \quad \lambda+2 \mu>0
$$

Simpson and Spector [4] treat the strong ellipticity condition for isotropic nonlinearly elastic materials. They establish necessary and sufficient conditions for the strong ellipticity of the equations governing an isotropic (compressible) nonlinearly elastic material at equilibrium. The main tool in their analysis consists in a representation theorem for copositive matrices. A review on strong ellipticity for isotropic nonlinearly elastic materials is given by Dacorogna [5].

Concerning the strong ellipticity for the class of anisotropic linearly elastic materials very few is known. A notable exception is the investigation given by Payton [6] and the recent results by Padovani [7], Merodio and Ogden [8] and Chiriţă [9] who examine the strong ellipticity for transversely isotropic linearly elastic solids. In terms of the elastic constants $c_{11}, c_{33}, c_{55}, c_{12}$ and $c_{13}$ of the transversely isotropic linearly elastic solid, the strong ellipticity condition is equivalent to the following inequalities

$$
\begin{gathered}
c_{11}>0, \quad c_{33}>0, \quad c_{55}>0, \quad c_{11}>c_{12}, \\
\left|c_{13}+c_{55}\right|<c_{55}+\sqrt{c_{11} c_{33}} .
\end{gathered}
$$

We recall that equivalence with respect to the restrictions that material symmetry places on the elasticity tensor for 32 crystal classes provides the following distinct situations (see Gurtin [1, pp. 87-89]):

(i) Triclinic system (21 elasticities);

(ii) Monoclinic system (all classes), (13 elasticities);

(iii) Rhombic system (all classes), (nine elasticities);

(iv) Tetragonal system (tetragonal-disphenoidal, tetragonal-pyramidal, tetragonal-dipyramidal), (seven elasticities); 
(v) Tetragonal system (tetragonal-scalenohedral, ditetragonal-pyramidal, tetragonal-trapezohedral, ditetragonal-dipyramidal), (six elasticities);

(vi) Cubic system (tetartoidal, diploidal, hexatetrahedral, gyroidal, hexoctahedral), (three elasticities);

(vii) Hexagonal system (trigonal-pyramidal, rhombohedral), (seven elasticities);

(viii) Hexagonal system (ditrigonal-pyramidal, trigonal-trapezohedral, hexagonal-scalenohedral), (six elasticities);

(ix) Hexagonal system (trigonal-dipyramidal, hexagonal-pyramidal, hexagonaldipyramidal, ditrigonal-dipyramidal, dihexagonal-pyramidal, hexagonaltrapezohedral, dihexagonal-dipyramidal, i.e., the hexagonal division), (five elasticities).

The form of the elasticity tensor imposed by the material symmetry requirements in the last case above is identical with that imposed by the transverse isotropy and this is the only anisotropic situation where necessary and sufficient conditions for strong ellipticity are known (see [6-9]).

In what follows we provide necessary and sufficient conditions equivalent to strong ellipticity that cover anisotropic materials in all classes of the rhombic system, i.e. the case (iii) above, a part of the tetragonal system covered by classes in (v) and all classes of the cubic system.

Our main results show that for rhombic materials and, in particular, more symmetric situations, strong ellipticity restricts some combinations among the classical elastic coefficients to take values inside a domain whose boundary has a simple algebraic form.

The paper is organized as follows: the second section provides statements of all results but the proof of Theorem 1, which solves the problem for the rhombic system is presented in detail in Section 3. In Section 4 we specialize the general results of Section 3 in order to cover the above mentioned symmetry classes. We end the paper with some general comments and remarks concerning various possible extensions of our main results and their intrinsic difficulties.

\section{Problem Setting and Main Results}

Throughout this paper we shall discuss the strong ellipticity condition (1.2) for the rhombic system and for all other more symmetric systems. In the present section we shall write the form of the strong ellipticity condition for each system under discussion and then we shall state the restrictions that it places on the corresponding elastic coefficients.

\subsection{Strong Ellipticity for the Rhombic System}

Let us consider the class of rhombic elastic materials with the group $\mathcal{C}_{3}$ generated by $\mathbf{R}_{\mathbf{e}_{3}}^{\pi}, \mathbf{R}_{\mathbf{e}_{2}}^{\pi}$ (here $\mathbf{R}_{\mathbf{e}}^{\theta}$ is the orthogonal tensor corresponding to a right-handed rotation through the angle $\theta, 0<\theta<2 \pi$, about an axis in the direction of the unit vector $\mathbf{e}$ ). 
According to Gurtin [1, p. 88] such a class of materials is characterized by the conditions

$$
\begin{aligned}
& C_{1123}=C_{1131}=C_{1112}=C_{2223}=C_{2231}=C_{2212}=0, \\
& C_{3323}=C_{3331}=C_{3312}=C_{2331}=C_{2312}=C_{3112}=0 .
\end{aligned}
$$

For convenience, we shall introduce a standard notation in linear elasticity, in order to denote the only non-zero components of the elasticity tensor as follows

$$
\begin{array}{r}
c_{11}=C_{1111}, c_{22}=C_{2222}, c_{33}=C_{3333}, c_{12}=C_{1122}, c_{23}=C_{2233}, \\
c_{31}=C_{3311}, c_{44}=C_{2323}, c_{55}=C_{1313}, c_{66}=C_{1212} .
\end{array}
$$

Using the relations (2.1) and (2.2), for a rhombic linearly elastic material the strong ellipticity condition (1.2) becomes

$$
\begin{aligned}
c_{11} n_{1}^{2} m_{1}^{2} & +c_{22} n_{2}^{2} m_{2}^{2}+c_{66}\left(n_{1} m_{2}+n_{2} m_{1}\right)^{2}+c_{33} n_{3}^{2} m_{3}^{2}+2 c_{12} n_{1} m_{1} n_{2} m_{2}+2 c_{13} n_{1} m_{1} n_{3} m_{3}+ \\
& +2 c_{23} n_{2} m_{2} n_{3} m_{3}+c_{44}\left(n_{3} m_{2}+n_{2} m_{3}\right)^{2}+c_{55}\left(n_{3} m_{1}+n_{1} m_{3}\right)^{2}>0
\end{aligned}
$$

for all non-zero vectors $\mathbf{m}=\left(m_{1}, m_{2}, m_{3}\right)$ and $\mathbf{n}=\left(n_{1}, n_{2}, n_{3}\right)$.

The following theorem provides restrictions on the elastic coefficients equivalent to strong ellipticity condition for all classes in the rhombic system.

Theorem 1 Suppose that the elastic material is rhombic $\left(\mathcal{C}_{3}\right.$-symmetry) with the group $\mathcal{C}_{3}$ generated by $\mathbf{R}_{\mathbf{e}_{3}}^{\pi}, \mathbf{R}_{\mathbf{e}_{2}}^{\pi}$. Then the elasticity tensor is strongly elliptic if and only if the following conditions are satisfied

$$
\begin{aligned}
& c_{11}>0, \quad c_{22}>0, \quad c_{33}>0, \quad c_{44}>0, \quad c_{55}>0, \quad c_{66}>0, \\
&-2 c_{66}+\varkappa_{3}^{i} \sqrt{c_{11} c_{22}}<c_{12}<\varkappa_{3}^{s} \sqrt{c_{11} c_{22}}, \\
&-2 c_{44}+\varkappa_{1}^{i} \sqrt{c_{22} c_{33}}<c_{23}<\varkappa_{1}^{s} \sqrt{c_{22} c_{33}}, \\
&-2 c_{55}+\varkappa_{2}^{i} \sqrt{c_{11} c_{33}}<c_{13}<\varkappa_{2}^{s} \sqrt{c_{11} c_{33}},
\end{aligned}
$$

where $\left(\varkappa_{1}^{i}, \varkappa_{1}^{s}\right),\left(\varkappa_{2}^{i}, \varkappa_{2}^{s}\right)$ and $\left(\varkappa_{3}^{i}, \varkappa_{3}^{s}\right)$ are solutions with respect to $x, y$ and $z$ of the equation

$$
S(x, y, z) \equiv x^{2}+y^{2}+z^{2}-2 x y z-1=0,
$$

satisfying $|x|<1,|y|<1,|z|<1$ and $x \in\left\{\frac{c_{23}}{\sqrt{c_{22} c_{33}}}, \frac{c_{23}+2 c_{44}}{\sqrt{c_{22} c_{33}}}\right\}, y \in\left\{\frac{c_{13}}{\sqrt{c_{11} c_{33}}}, \frac{c_{13}+2 c_{55}}{\sqrt{c_{11} c_{33}}}\right\}$, $z \in\left\{\frac{c_{12}}{\sqrt{c_{11} c_{22}}}, \frac{c_{12}+2 c_{66}}{\sqrt{c_{11} c_{22}}}\right\}$.

A direct consequence of our subsequent analysis is the following result.

Corollary The strong ellipticity condition (2.3) holds if and only if the relation (2.4) is satisfied and all points $P(x, y, z)$, with coordinates $x \in\left\{\frac{c_{23}}{\sqrt{c_{22} c_{33}}}, \frac{c_{23}+2 c_{44}}{\sqrt{c_{22} c_{33}}}\right\}$, $y \in\left\{\frac{c_{13}}{\sqrt{c_{11} c_{33}}}, \frac{c_{13}+2 c_{55}}{\sqrt{c_{11} c_{33}}}\right\}, z \in\left\{\frac{c_{12}}{\sqrt{c_{11} c_{22}}}, \frac{c_{12}+2 c_{66}}{\sqrt{c_{11} c_{22}}}\right\}$, lie inside the region limited by the surface $S(x, y, z) \equiv x^{2}+y^{2}+z^{2}-2 x y z-1=0, \quad|x|<1,|y|<1,|z|<1$. 
Fig. 1 Surface $S(x, y, z)$ in the cube $|x| \leq 1,|y| \leq 1,|z| \leq 1$

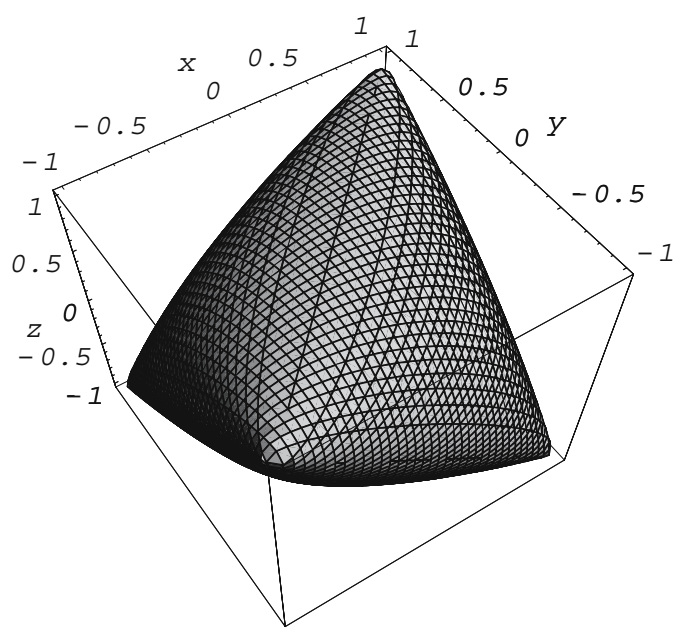

The part of the surface $S(x, y, z)$ situated in the cube $|x| \leq 1,|y| \leq 1,|z| \leq 1$ is given in Fig. 1, while the Fig. 2 gives an image of the same surface in the extended cube $|x| \leq 2,|y| \leq 2,|z| \leq 2$.

\subsection{Strong Ellipticity for the Hexagonal Division and Transverse Isotropy}

The restrictions placed on the elasticity tensor by material symmetry in seven crystal classes of the hexagonal systems are identical with those imposed by transverse isotropy (see Gurtin [1, p. 89]). The explicit restrictions on elasticities for symmetry

Fig. 2 Surface $S(x, y, z)$ in the cube $|x| \leq 2,|y| \leq 2,|z| \leq 2$

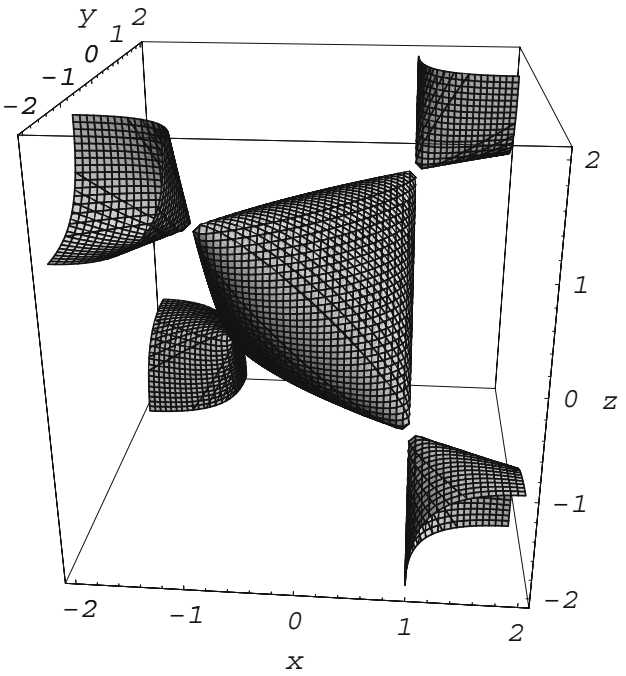

Springer 
groups $\mathcal{C}_{10}$ generated by $\mathbf{R}_{\mathbf{e}_{3}}^{\pi / 3}, \mathcal{C}_{11}$ generated by $\mathbf{R}_{\mathbf{e}_{3}}^{\pi / 3}, \mathbf{R}_{\mathbf{e}_{2}}^{\pi}$ and transverse isotropy $\mathcal{C}_{12}$ generated by $\mathbf{R}_{\mathbf{e}_{3}}^{\varphi}, 0<\varphi<2 \pi$ are characterized by the relations (2.1) and (2.2) and

$$
c_{22}=c_{11}, \quad c_{23}=c_{13}, \quad c_{44}=c_{55}, \quad c_{66}=\frac{1}{2}\left(c_{11}-c_{12}\right)
$$

and the strong ellipticity condition (1.2) becomes

$$
\begin{gathered}
c_{66}\left(n_{1} m_{2}-n_{2} m_{1}\right)^{2}+c_{11}\left(n_{1} m_{1}+n_{2} m_{2}\right)^{2}+2\left(c_{13}+c_{55}\right)\left(n_{1} m_{1}+n_{2} m_{2}\right) n_{3} m_{3}+ \\
+c_{33} n_{3}^{2} m_{3}^{2}+c_{55}\left(n_{3}^{2} m_{1}^{2}+n_{1}^{2} m_{3}^{2}+n_{3}^{2} m_{2}^{2}+n_{2}^{2} m_{3}^{2}\right)>0 .
\end{gathered}
$$

Theorem 2 The strong ellipticity condition (2.8) for a transversely isotropic elastic material is equivalent with the following conditions

$$
c_{11}>0, \quad c_{33}>0, \quad c_{55}>0, \quad c_{11}>c_{12}, \quad\left|c_{13}+c_{55}\right|<c_{55}+\sqrt{c_{11} c_{33}} .
$$

These last conditions have been previously established by Payton [6], Padovani [7], Merodio and Ogden [8] and Chiriţă [9].

\subsection{Strong Ellipticity for the Tetragonal System}

Restrictions placed by symmetries in the seven crystal classes of the tetragonal system are covered by two distinct situations. Three classes, i.e., tetragonal-disphenoidal, tetragonal-pyramidal, tetragonal-dipyramidal provide an elasticity tensor with seven elastic coefficients while the others, i.e. tetragonal-scalenohedral, ditetragonalpyramidal, tetragonal-trapezohedral, ditetragonal-dipyramidal provide an elasticity tensor with six elastic coefficients. This subsection cover the last situation.

The tetragonal system $\mathcal{C}_{5}$ generated by $\mathbf{R}_{\mathbf{e}_{3}}^{\pi / 2}, \mathbf{R}_{\mathbf{e}_{1}}^{\pi}$ is characterized by the relations (2.1) and (2.2) and

$$
c_{22}=c_{11}, \quad c_{23}=c_{13}, \quad c_{44}=c_{55} .
$$

Moreover, the strong ellipticity condition (1.2) becomes

$$
\begin{gathered}
c_{11}\left(n_{1} m_{1}+n_{2} m_{2}\right)^{2}+c_{66}\left(n_{1} m_{2}+n_{2} m_{1}\right)^{2}+c_{33} n_{3}^{2} m_{3}^{2}+2\left(c_{12}-c_{11}\right) n_{1} m_{1} n_{2} m_{2}+ \\
+2 c_{13}\left(n_{1} m_{1}+n_{2} m_{2}\right) n_{3} m_{3}+c_{55}\left[\left(n_{3} m_{2}+n_{2} m_{3}\right)^{2}+\left(n_{3} m_{1}+n_{1} m_{3}\right)^{2}\right]>0 .
\end{gathered}
$$

Theorem 3 The strong ellipticity condition (2.11) for the tetragonal system $\mathcal{C}_{5}$ generated by $\mathbf{R}_{\mathbf{e}_{3}}^{\pi / 2}, \mathbf{R}_{\mathbf{e}_{1}}^{\pi}$ is equivalent with the following conditions

$$
\begin{gathered}
c_{11}>0, \quad c_{33}>0, \quad c_{55}>0, \quad c_{66}>0, \\
-2 c_{66}+\varkappa_{3}^{i} c_{11}<c_{12}<\varkappa_{3}^{s} c_{11}, \\
-2 c_{55}+\varkappa_{2}^{i} \sqrt{c_{11} c_{33}}<c_{13}<\varkappa_{2}^{s} \sqrt{c_{11} c_{33}},
\end{gathered}
$$


where

$$
\begin{array}{ll}
\varkappa_{3}^{i}=2 x^{2}-1, \quad \varkappa_{3}^{s}=1, \quad \text { for } x \in(-1,1), \\
\varkappa_{2}^{i}=-\sqrt{\frac{1+z}{2}}, \quad \varkappa_{2}^{s}=\sqrt{\frac{1+z}{2}}, \quad \text { for } z \in(-1,1) .
\end{array}
$$

The strong ellipticity region is illustrated in Fig. 3, with $x=\frac{c_{13}}{\sqrt{c_{11} c_{33}}}$ or $x=\frac{c_{13}+2 c_{55}}{\sqrt{c_{11} c_{33}}}$ and $z=\frac{c_{12}}{\sqrt{c_{11} c_{22}}}$ or $z=\frac{c_{12}+2 c_{66}}{\sqrt{c_{11} c_{22}}}$.

\subsection{Strong Ellipticity for the Cubic System}

Restrictions imposed by symmetries in all the five classes of the cubic system provide three elasticities. The cubic system $\mathcal{C}_{6}$ generated by $\mathbf{R}_{\mathbf{e}_{1}}^{\pi}, \mathbf{R}_{\mathbf{e}_{2}}^{\pi}, \mathbf{R}_{\mathbf{q}}^{2 \pi / 3}$, $\mathbf{q}=\sqrt{\frac{1}{3}}\left(\mathbf{e}_{1}+\mathbf{e}_{2}+\mathbf{e}_{3}\right)$ and $\mathcal{C}_{7}$ generated by $\mathbf{R}_{\mathbf{e}_{1}}^{\pi / 2}, \mathbf{R}_{\mathbf{e}_{2}}^{\pi / 2}, \mathbf{R}_{\mathbf{e}_{3}}^{\pi / 2}$ (with 3 elasticities) is characterized by the relations (2.1) and (2.2) and

$$
c_{11}=c_{22}=c_{33}, \quad c_{12}=c_{23}=c_{13}, \quad c_{44}=c_{55}=c_{66}
$$

and the strong ellipticity condition (1.2) becomes

$$
\begin{aligned}
& c_{11}\left(n_{1} m_{1}+n_{2} m_{2}+n_{3} m_{3}\right)^{2}+2\left(c_{12}-c_{11}\right)\left(n_{1} m_{1} n_{2} m_{2}+n_{2} m_{2} n_{3} m_{3}+n_{3} m_{3} n_{1} m_{1}\right)+ \\
& \quad+c_{66}\left[\left(n_{1} m_{2}+n_{2} m_{1}\right)^{2}+\left(n_{3} m_{2}+n_{2} m_{3}\right)^{2}+\left(n_{3} m_{1}+n_{1} m_{3}\right)^{2}\right]>0 .
\end{aligned}
$$

Fig. 3 Strong ellipticity region for tetragonal system

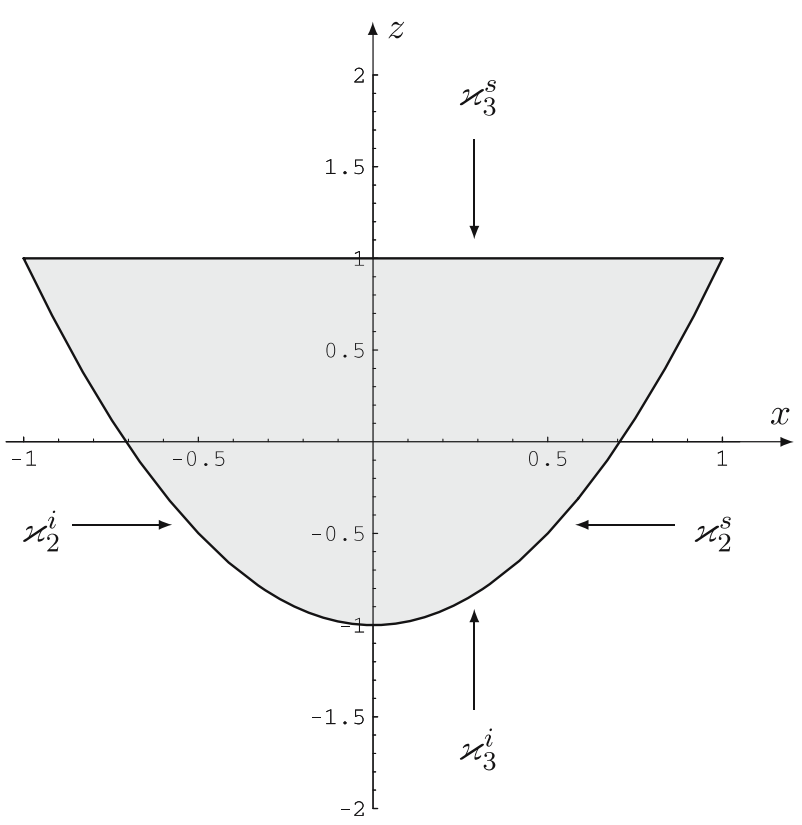


Theorem 4 The strong ellipticity condition (2.18) for a cubic system is equivalent with the following conditions

$$
c_{11}>0, \quad c_{66}>0, \quad\left|c_{12}+c_{66}\right|<c_{11}+c_{66} .
$$

\section{Proof of the Theorem 1}

This section contains the proof for our main result described by the Theorem 1 . We start considering the strong ellipticity condition (2.3) as a quadratic in one of the variables $m_{1}, m_{2}, m_{3}, n_{1}, n_{2}, n_{3}$. Thus, we rewrite the relation (2.3) in the following form

$$
\begin{aligned}
\left(c_{55} m_{1}^{2}\right. & \left.+c_{44} m_{2}^{2}+c_{33} m_{3}^{2}\right) n_{3}^{2}+2\left[\left(c_{13}+c_{55}\right) n_{1} m_{1}+\left(c_{23}+c_{44}\right) n_{2} m_{2}\right] m_{3} n_{3}+ \\
& +c_{11} n_{1}^{2} m_{1}^{2}+c_{22} n_{2}^{2} m_{2}^{2}+c_{66} n_{1}^{2} m_{2}^{2}+c_{66} n_{2}^{2} m_{1}^{2}+2\left(c_{12}+c_{66}\right) n_{1} m_{1} n_{2} m_{2}+ \\
& +\left(c_{55} n_{1}^{2}+c_{44} n_{2}^{2}\right) m_{3}^{2}>0 .
\end{aligned}
$$

Regarding (3.1) as a quadratic in $n_{3} \in \mathbb{R}$, we deduce

$$
\begin{aligned}
c_{55} m_{1}^{2} & +c_{44} m_{2}^{2}+c_{33} m_{3}^{2}>0 \quad \text { for all } \mathbf{m}=\left(m_{1}, m_{2}, m_{3}\right) \neq \mathbf{0}, \\
c_{11} n_{1}^{2} m_{1}^{2} & +c_{22} n_{2}^{2} m_{2}^{2}+c_{66} n_{1}^{2} m_{2}^{2}+c_{66} n_{2}^{2} m_{1}^{2}+2\left(c_{12}+c_{66}\right) n_{1} m_{1} n_{2} m_{2}+ \\
& +\left(c_{55} n_{1}^{2}+c_{44} n_{2}^{2}\right) m_{3}^{2}>0
\end{aligned}
$$

and the discriminant has to be strictly negative for all non-zero vectors $\mathbf{m}=$ $\left(m_{1}, m_{2}, m_{3}\right)$ and $\mathbf{n}=\left(n_{1}, n_{2}, n_{3}\right)$.

As a direct consequence, from the relation (3.2) we obtain that

$$
c_{33}>0, \quad c_{44}>0, \quad c_{55}>0,
$$

while the relation (3.3) implies

$$
c_{11} n_{1}^{2} m_{1}^{2}+c_{22} n_{2}^{2} m_{2}^{2}+c_{66} n_{1}^{2} m_{2}^{2}+c_{66} n_{2}^{2} m_{1}^{2}+2\left(c_{12}+c_{66}\right) n_{1} m_{1} n_{2} m_{2}>0,
$$

that is

$$
\left(c_{11} m_{1}^{2}+c_{66} m_{2}^{2}\right) n_{1}^{2}+2\left(c_{12}+c_{66}\right) m_{1} m_{2} n_{2} n_{1}+\left(c_{66} m_{1}^{2}+c_{22} m_{2}^{2}\right) n_{2}^{2}>0,
$$

for all non-zero vectors $\mathbf{N}=\left(n_{1}, n_{2}\right), \mathbf{M}=\left(m_{1}, m_{2}\right)$. Considering (3.6) as a quadratic in $n_{1} \in \mathbb{R}$, we deduce

$$
\begin{aligned}
& c_{11} m_{1}^{2}+c_{66} m_{2}^{2}>0, \quad c_{66} m_{1}^{2}+c_{22} m_{2}^{2}>0 \quad \text { for all } \quad\left(m_{1}, m_{2}\right) \neq \mathbf{0}, \\
& \left(c_{12}+c_{66}\right)^{2} m_{1}^{2} m_{2}^{2}<\left(c_{11} m_{1}^{2}+c_{66} m_{2}^{2}\right)\left(c_{66} m_{1}^{2}+c_{22} m_{2}^{2}\right) \text { for all }\left(m_{1}, m_{2}\right) \neq \mathbf{0} .
\end{aligned}
$$

Consequently, the relation (3.7) implies

$$
c_{11}>0, \quad c_{22}>0, \quad c_{66}>0,
$$


and the relation (3.8) becomes

$$
c_{11} c_{66} m_{1}^{4}+\left[c_{11} c_{22}+c_{66}^{2}-\left(c_{12}+c_{66}\right)^{2}\right] m_{1}^{2} m_{2}^{2}+c_{22} c_{66} m_{2}^{4}>0,
$$

that is,

$$
c_{66}\left(\sqrt{c_{11}} m_{1}^{2}-\sqrt{c_{22}} m_{2}^{2}\right)^{2}+\left[\left(c_{66}+\sqrt{c_{11} c_{22}}\right)^{2}-\left(c_{12}+c_{66}\right)^{2}\right] m_{1}^{2} m_{2}^{2}>0 .
$$

Therefore, setting $\sqrt{c_{11}} m_{1}^{2}=\sqrt{c_{22}} m_{2}^{2}$ in (3.11), we obtain

$$
\left(c_{66}+\sqrt{c_{11} c_{22}}\right)^{2}-\left(c_{12}+c_{66}\right)^{2}>0
$$

and hence we have

$$
\left|c_{12}+c_{66}\right|<c_{66}+\sqrt{c_{11} c_{22}} .
$$

We now return to the equation (3.1) and note that the condition expressing the negativeness of the discriminant gives

$$
\begin{aligned}
& {\left[\left(c_{13}+c_{55}\right) n_{1} m_{1}+\left(c_{23}+c_{44}\right) n_{2} m_{2}\right]^{2} m_{3}^{2}<\left(c_{55} m_{1}^{2}+c_{44} m_{2}^{2}+c_{33} m_{3}^{2}\right) } . \\
& \cdot\left[\left(c_{55} n_{1}^{2}+c_{44} n_{2}^{2}\right) m_{3}^{2}+c_{11} n_{1}^{2} m_{1}^{2}+c_{22} n_{2}^{2} m_{2}^{2}+c_{66} n_{1}^{2} m_{2}^{2}+\right. \\
&\left.+c_{66} n_{2}^{2} m_{1}^{2}+2\left(c_{12}+c_{66}\right) n_{1} m_{1} n_{2} m_{2}\right]
\end{aligned}
$$

and hence

$$
\begin{aligned}
c_{33}\left(c_{55} n_{1}^{2}\right. & \left.+c_{44} n_{2}^{2}\right) m_{3}^{4}+\left\{\left(c_{55} m_{1}^{2}+c_{44} m_{2}^{2}\right)\left(c_{55} n_{1}^{2}+c_{44} n_{2}^{2}\right)+c_{33}\left[c_{11} n_{1}^{2} m_{1}^{2}+\right.\right. \\
& \left.+c_{22} n_{2}^{2} m_{2}^{2}+c_{66} n_{1}^{2} m_{2}^{2}+c_{66} n_{2}^{2} m_{1}^{2}+2\left(c_{12}+c_{66}\right) n_{1} m_{1} n_{2} m_{2}\right]- \\
& \left.-\left[\left(c_{13}+c_{55}\right) n_{1} m_{1}+\left(c_{23}+c_{44}\right) n_{2} m_{2}\right]^{2}\right\} m_{3}^{2}+\left(c_{55} m_{1}^{2}+c_{44} m_{2}^{2}\right) . \\
\cdot & {\left[c_{11} n_{1}^{2} m_{1}^{2}+c_{22} n_{2}^{2} m_{2}^{2}+c_{66} n_{1}^{2} m_{2}^{2}+c_{66} n_{2}^{2} m_{1}^{2}+2\left(c_{12}+c_{66}\right) n_{1} m_{1} n_{2} m_{2}\right]>0 . }
\end{aligned}
$$

Therefore, if we set

$$
\begin{aligned}
m_{3}^{2}= & \frac{1}{\sqrt{c_{33}}} \sqrt{\frac{c_{55} m_{1}^{2}+c_{44} m_{2}^{2}}{c_{55} n_{1}^{2}+c_{44} n_{2}^{2}}} . \\
& \cdot \sqrt{c_{11} n_{1}^{2} m_{1}^{2}+c_{22} n_{2}^{2} m_{2}^{2}+c_{66} n_{1}^{2} m_{2}^{2}+c_{66} n_{2}^{2} m_{1}^{2}+2\left(c_{12}+c_{66}\right) n_{1} m_{1} n_{2} m_{2}},
\end{aligned}
$$

into relation (3.15), then we deduce

$$
\begin{aligned}
& \left|\left(c_{13}+c_{55}\right) n_{1} m_{1}+\left(c_{23}+c_{44}\right) n_{2} m_{2}\right|<\sqrt{\left(c_{55} m_{1}^{2}+c_{44} m_{2}^{2}\right)\left(c_{55} n_{1}^{2}+c_{44} n_{2}^{2}\right)}+ \\
& \quad+\sqrt{c_{33}} \sqrt{c_{11} n_{1}^{2} m_{1}^{2}+c_{22} n_{2}^{2} m_{2}^{2}+c_{66} n_{1}^{2} m_{2}^{2}+c_{66} n_{2}^{2} m_{1}^{2}+2\left(c_{12}+c_{66}\right) n_{1} m_{1} n_{2} m_{2}},
\end{aligned}
$$

for all non-zero vectors $\mathbf{N}=\left(n_{1}, n_{2}\right), \mathbf{M}=\left(m_{1}, m_{2}\right)$.

Next step is the study of restrictions placed on the elastic coefficients by the inequality (3.17). We start noting that the explicit expression of the term on the left 
hand side of the relation (3.17) depends on the sign of the terms $c_{13}+c_{55}, c_{23}+c_{44}$, $n_{1} m_{1}$ and $n_{2} m_{2}$. For this reason we rewrite it in an equivalent form more tractable in the subsequent analysis.

Let us assume that the terms $c_{13}+c_{55}$ and $c_{23}+c_{44}$ are prescribed. Then the explicit expression of the term on the left hand side of the relation (3.17) depends on the sign of the terms $n_{1} m_{1}$ and $n_{2} m_{2}$ only. In fact, when $n_{1} m_{1}$ and $n_{2} m_{2}$ have the same sign the relation (3.17) becomes

$$
\begin{aligned}
& \left|\left(c_{13}+c_{55}\right)\right| n_{1} m_{1}\left|+\left(c_{23}+c_{44}\right)\right| n_{2} m_{2}||<\sqrt{\left(c_{55} m_{1}^{2}+c_{44} m_{2}^{2}\right)\left(c_{55} n_{1}^{2}+c_{44} n_{2}^{2}\right)}+ \\
& \quad+\sqrt{c_{33}} \sqrt{c_{11} n_{1}^{2} m_{1}^{2}+c_{22} n_{2}^{2} m_{2}^{2}+c_{66} n_{1}^{2} m_{2}^{2}+c_{66} n_{2}^{2} m_{1}^{2}+2\left(c_{12}+c_{66}\right)\left|n_{1} m_{1}\right|\left|n_{2} m_{2}\right|}
\end{aligned}
$$

while when $\left(n_{1} m_{1}\right) \cdot\left(n_{2} m_{2}\right)<0$ we have

$$
\begin{aligned}
& \left|-\left(c_{13}+c_{55}\right)\right| n_{1} m_{1}\left|+\left(c_{23}+c_{44}\right)\right| n_{2} m_{2}||<\sqrt{\left(c_{55} m_{1}^{2}+c_{44} m_{2}^{2}\right)\left(c_{55} n_{1}^{2}+c_{44} n_{2}^{2}\right)}+ \\
& \quad+\sqrt{c_{33}} \sqrt{c_{11} n_{1}^{2} m_{1}^{2}+c_{22} n_{2}^{2} m_{2}^{2}+c_{66} n_{1}^{2} m_{2}^{2}+c_{66} n_{2}^{2} m_{1}^{2}-2\left(c_{12}+c_{66}\right)\left|n_{1} m_{1}\right|\left|n_{2} m_{2}\right|}
\end{aligned}
$$

for all non-zero vectors $\mathbf{N}=\left(n_{1}, n_{2}\right), \mathbf{M}=\left(m_{1}, m_{2}\right)$.

Further, we can prove that the relation (3.18) is equivalent with the following relation

$$
\begin{gathered}
\left|\left(c_{13}+c_{55}\right) m_{1}^{2}+\left(c_{23}+c_{44}\right) m_{2}^{2}\right|<c_{55} m_{1}^{2}+c_{44} m_{2}^{2}+ \\
+\sqrt{c_{33}} \sqrt{c_{11} m_{1}^{4}+c_{22} m_{2}^{4}+2\left(c_{12}+2 c_{66}\right) m_{1}^{2} m_{2}^{2}} \text {, for all }\left(m_{1}, m_{2}\right) \neq \mathbf{0},
\end{gathered}
$$

and the relation (3.19) is equivalent with the following one

$$
\begin{gathered}
\left|-\left(c_{13}+c_{55}\right) m_{1}^{2}+\left(c_{23}+c_{44}\right) m_{2}^{2}\right|<c_{55} m_{1}^{2}+c_{44} m_{2}^{2}+ \\
+\sqrt{c_{33}} \sqrt{c_{11} m_{1}^{4}+c_{22} m_{2}^{4}-2 c_{12} m_{1}^{2} m_{2}^{2}}, \text { for all }\left(m_{1}, m_{2}\right) \neq \mathbf{0} .
\end{gathered}
$$

Let us first assume that the inequality (3.18) holds for all non-zero vectors $\left(n_{1}, n_{2}\right)$, $\left(m_{1}, m_{2}\right)$. If we set $n_{1}=\alpha m_{1}, n_{2}=\alpha m_{2}$, with $\alpha \in \mathbb{R}$, into relation (3.18), then we obtain the relation (3.20). In a similar manner we can prove that (3.19) implies (3.21).

Conversely, assume that the relation (3.20) holds. If we set $m_{1} \rightarrow \sqrt{\left|n_{1} m_{1}\right|}$ and $m_{2} \rightarrow \sqrt{\left|n_{2} m_{2}\right|}$ into (3.20), we obtain

$$
\begin{aligned}
& \left|\left(c_{13}+c_{55}\right)\right| n_{1} m_{1}\left|+\left(c_{23}+c_{44}\right)\right| n_{2} m_{2}||<c_{55}\left|n_{1} m_{1}\right|+c_{44}\left|n_{2} m_{2}\right|+ \\
& \quad+\sqrt{c_{33}} \sqrt{c_{11} n_{1}^{2} m_{1}^{2}+c_{22} n_{2}^{2} m_{2}^{2}+2\left(c_{12}+c_{66}\right)\left|n_{1} m_{1} n_{2} m_{2}\right|+2 c_{66}\left|n_{1} m_{2}\right|\left|n_{2} m_{1}\right|} \\
& \text { Springer }
\end{aligned}
$$


for all non-zero vectors $\left(n_{1}, n_{2}\right),\left(m_{1}, m_{2}\right)$. Further, we note that by means of the relation (3.4) and the Schwarz inequality, we have

$$
c_{55}\left|n_{1} m_{1}\right|+c_{44}\left|n_{2} m_{2}\right| \leq \sqrt{c_{55} m_{1}^{2}+c_{44} m_{2}^{2}} \sqrt{c_{55} n_{1}^{2}+c_{44} n_{2}^{2}}
$$

while the relation (3.9) and the arithmetic-geometric mean inequality furnish

$$
2 c_{66}\left|n_{1} m_{2}\right|\left|n_{2} m_{1}\right| \leq c_{66} n_{1}^{2} m_{2}^{2}+c_{66} n_{2}^{2} m_{1}^{2} .
$$

Thus, when used in (3.22), the relations (3.23) and (3.24) give the relation (3.18).

Assuming now $m_{1} \rightarrow \sqrt{\left|n_{1} m_{1}\right|}$ and $m_{2} \rightarrow \sqrt{\left|n_{2} m_{2}\right|}$ into relation (3.21), we obtain

$$
\begin{aligned}
& \left|-\left(c_{13}+c_{55}\right)\right| n_{1} m_{1}\left|+\left(c_{23}+c_{44}\right)\right| n_{2} m_{2}||<c_{55}\left|n_{1} m_{1}\right|+c_{44}\left|n_{2} m_{2}\right|+ \\
& \quad+\sqrt{c_{33}} \sqrt{c_{11} n_{1}^{2} m_{1}^{2}+c_{22} n_{2}^{2} m_{2}^{2}-2\left(c_{12}+c_{66}\right)\left|n_{1} m_{1} n_{2} m_{2}\right|+2 c_{66}\left|n_{1} m_{2}\right|\left|n_{2} m_{1}\right|}
\end{aligned}
$$

for all non-zero vectors $\left(n_{1}, n_{2}\right),\left(m_{1}, m_{2}\right)$. When we use the relations (3.23) and (3.24) into relation (3.25) we are led to the relation (3.19).

On this basis we will proceed in what follows to find necessary and sufficient conditions in order to satisfy the relations (3.20) and (3.21).

For later convenience we note that if we set $m_{2}=0$ into relation (3.20) then we obtain

$$
\left|c_{13}+c_{55}\right|<c_{55}+\sqrt{c_{11} c_{33}},
$$

while, for $m_{1}=0$, the relation (3.21) furnishes

$$
\left|c_{23}+c_{44}\right|<c_{44}+\sqrt{c_{22} c_{33}} \text {. }
$$

At this point we note that the restrictions (3.13), (3.26) and (3.27) are equivalent to the following estimates

$$
\begin{array}{ll}
-1<\frac{c_{12}+2 c_{66}}{\sqrt{c_{11} c_{22}}}, & \frac{c_{12}}{\sqrt{c_{11} c_{22}}}<1, \\
-1<\frac{c_{23}+2 c_{44}}{\sqrt{c_{22} c_{33}}}, & \frac{c_{23}}{\sqrt{c_{22} c_{33}}}<1, \\
-1<\frac{c_{13}+2 c_{55}}{\sqrt{c_{11} c_{33}}}, & \frac{c_{13}}{\sqrt{c_{11} c_{33}}}<1 .
\end{array}
$$

We have now all the preliminary material to establish the restrictions implied by the inequality (3.17). The discussion is guided by the sign of the two terms entering the left hand side of the relations (3.20) and (3.21), that is the sign of the coefficients $c_{13}+c_{55}$ and $c_{23}+c_{44}$. As we will see later, when $c_{12}, c_{13}$ and $c_{23}$ vary according with the restrictions (3.28), the relations (3.20) and (3.21) furnish improved lower bounds for $\frac{c_{12}+2 c_{66}}{\sqrt{c_{11} c_{22}}}, \frac{c_{23}+2 c_{44}}{\sqrt{c_{22} c_{33}}}$ and $\frac{c_{13}+2 c_{55}}{\sqrt{c_{11} c_{33}}}$ and upper bounds for $\frac{c_{12}}{\sqrt{c_{11} c_{22}}}$, $\frac{c_{23}}{\sqrt{c_{22} c_{33}}}$ and $\frac{c_{13}}{\sqrt{c_{11} c_{33}}}$. In fact, we will prove that all points $P(x, y, z)$, with coordinates $x \in\left\{\frac{c_{23}+2 c_{44}}{\sqrt{c_{22} c_{33}}}, \frac{c_{23}}{\sqrt{c_{22} c_{33}}}\right\}, y \in\left\{\frac{c_{13}+2 c_{55}}{\sqrt{c_{11} c_{33}}}, \frac{c_{13}}{\sqrt{c_{11} c_{33}}}\right\}, z \in\left\{\frac{c_{12}+2 c_{66}}{\sqrt{c_{11} c_{22}}}, \frac{c_{12}}{\sqrt{c_{11} c_{22}}}\right\}$, are situated inside of the region limited by the surface $S(x, y, z) \equiv x^{2}+y^{2}+z^{2}-2 x y z-1=0,(x, y, z) \in$ $(-1,1) \times(-1,1) \times(-1,1)$ (see Fig. 1). 
We have to develop our analysis of the relations (3.20) and (3.21) for the following cases: 1) $\left(c_{13}+c_{55}\right) \cdot\left(c_{23}+c_{44}\right)>0$ and 2$)\left(c_{13}+c_{55}\right) \cdot\left(c_{23}+c_{44}\right)<0$. When $c_{13}+$ $c_{55}=0$ or $c_{23}+c_{44}=0$ the discussion follows the same procedure.

\subsection{The Case $\left(c_{13}+c_{55}\right) \cdot\left(c_{23}+c_{44}\right)>0$}

Throughout this subsection we will assume that $\left(c_{13}+c_{55}\right) \cdot\left(c_{23}+c_{44}\right)>0$. For convenience we first study the case when

$$
c_{13}+c_{55}>0, \quad c_{23}+c_{44}>0,
$$

while the case $c_{13}+c_{55}<0, c_{23}+c_{44}<0$ will be discussed in short at the end of this subsection. We recall that the relations (3.26) and (3.27) are equivalent with

$$
-2 c_{55}-\sqrt{c_{11} c_{33}}<c_{13}<\sqrt{c_{11} c_{33}}, \quad-2 c_{44}-\sqrt{c_{22} c_{33}}<c_{23}<\sqrt{c_{22} c_{33}},
$$

and therefore, the case in discussion is characterized by

$$
-c_{55}<c_{13}<\sqrt{c_{11} c_{33}}, \quad-c_{44}<c_{23}<\sqrt{c_{22} c_{33}} \text {. }
$$

Under such hypothesis we will study the restrictions imposed upon the elastic coefficients by the relations (3.20) and (3.21).

\subsubsection{Analysis of Relation (3.20)}

In view of the assumption (3.31), relation (3.20) becomes

$$
c_{13} m_{1}^{2}+c_{23} m_{2}^{2}<\sqrt{c_{33}} \sqrt{c_{11} m_{1}^{4}+c_{22} m_{2}^{4}+2\left(c_{12}+2 c_{66}\right) m_{1}^{2} m_{2}^{2}} \text { for all }\left(m_{1}, m_{2}\right) \neq \mathbf{0} .
$$

In order to study the above inequality we have to consider the following four cases:

(1) $c_{13} \in\left(-c_{55}, 0\right]$ and $c_{23} \in\left(-c_{44}, 0\right]$;

(2) $c_{13} \in\left(0, \sqrt{c_{11} c_{33}}\right)$ and $c_{23} \in\left(0, \sqrt{c_{22} c_{33}}\right)$;

(3) $c_{13} \in\left(-c_{55}, 0\right]$ and $c_{23} \in\left(0, \sqrt{c_{22} c_{33}}\right)$;

(4) $c_{13} \in\left(0, \sqrt{c_{11} c_{33}}\right)$ and $c_{23} \in\left(-c_{44}, 0\right]$.

In case (1) inequality (3.32) is trivially satisfied. So we shall proceed to study the case (2). Then relation (3.32) implies

$$
c_{13}^{2} m_{1}^{4}+c_{23}^{2} m_{2}^{4}+2 c_{13} c_{23} m_{1}^{2} m_{2}^{2}<c_{11} c_{33} m_{1}^{4}+c_{22} c_{33} m_{2}^{4}+2 c_{33}\left(c_{12}+2 c_{66}\right) m_{1}^{2} m_{2}^{2}
$$

and hence we have

$$
\left(c_{11} c_{33}-c_{13}^{2}\right) m_{1}^{4}+\left(c_{22} c_{33}-c_{23}^{2}\right) m_{2}^{4}+2\left[c_{33}\left(c_{12}+2 c_{66}\right)-c_{13} c_{23}\right] m_{1}^{2} m_{2}^{2}>0 .
$$

The relation (3.34) is trivially satisfied when $c_{33}\left(c_{12}+2 c_{66}\right)-c_{13} c_{23} \geq 0$ so we shall assume that

$$
c_{33}\left(c_{12}+2 c_{66}\right)-c_{13} c_{23}<0 .
$$


Then we can write the relation (3.34) as

$$
\begin{gathered}
\left(\sqrt{c_{11} c_{33}-c_{13}^{2}} m_{1}^{2}-\sqrt{c_{22} c_{33}-c_{23}^{2}} m_{2}^{2}\right)^{2}+2\left[c_{33}\left(c_{12}+2 c_{66}\right)-c_{13} c_{23}+\right. \\
\left.+\sqrt{\left(c_{11} c_{33}-c_{13}^{2}\right)\left(c_{22} c_{33}-c_{23}^{2}\right)}\right] m_{1}^{2} m_{2}^{2}>0 \text { for all }\left(m_{1}, m_{2}\right) \neq \mathbf{0}
\end{gathered}
$$

Therefore, if we set

$$
m_{2}^{2}=\sqrt{\frac{c_{11} c_{33}-c_{13}^{2}}{c_{22} c_{33}-c_{23}^{2}}} m_{1}^{2}
$$

in (3.36), we deduce that

$$
\frac{c_{12}+2 c_{66}}{\sqrt{c_{11} c_{22}}}>\frac{c_{13}}{\sqrt{c_{11} c_{33}}} \frac{c_{23}}{\sqrt{c_{22} c_{33}}}-\sqrt{\left(1-\frac{c_{13}^{2}}{c_{11} c_{33}}\right)\left(1-\frac{c_{23}^{2}}{c_{22} c_{33}}\right)}>-1,
$$

provided the assumption (3.35) holds true. Thus, the relation (3.20) imposes a more severe restriction upon the term $\frac{c_{12}+2 c_{66}}{\sqrt{c_{11} c_{22}}}$ with respect to that described by the relation (3.28), in which we have only $\frac{c_{12}+2 c_{66}}{\sqrt{c_{11} c_{22}}}>-1$.

Let us consider the case (3). If we set $m_{2}=\beta m_{1}$, with $\beta \in \mathbb{R}$, into relation (3.32) then we can write

$$
c_{13}+c_{23} \beta^{2}<\sqrt{c_{33}} \sqrt{c_{11}+c_{22} \beta^{4}+2\left(c_{12}+2 c_{66}\right) \beta^{2}} \text { for all } \beta \in \mathbb{R} .
$$

From (3.13) we have

$$
-\sqrt{c_{11} c_{22}}<c_{12}+2 c_{66}<2 c_{66}+\sqrt{c_{11} c_{22}}
$$

so that if $c_{12}+2 c_{66} \in\left[0,2 c_{66}+\sqrt{c_{11} c_{22}}\right)$ then

$$
\begin{aligned}
c_{13}+c_{23} \beta^{2} & \leq c_{23} \beta^{2}=\sqrt{c_{23}^{2} \beta^{4}}<\sqrt{c_{22} c_{33} \beta^{4}}< \\
& <\sqrt{c_{11} c_{33}+c_{22} c_{33} \beta^{4}+2 c_{33}\left(c_{12}+2 c_{66}\right) \beta^{2}}
\end{aligned}
$$

and hence inequality (3.39) is trivially satisfied.

In what follows we shall discuss the case (3) under the assumption

$$
c_{12}+2 c_{66} \in\left(-\sqrt{c_{11} c_{22}}, 0\right) .
$$

We first observe that the relation (3.39) is trivially satisfied when $c_{13}+c_{23} \beta^{2} \leq 0$. Further, for $c_{13}+c_{23} \beta^{2}>0$ we set

$$
t=\sqrt{\beta^{2}+\frac{c_{13}}{c_{23}}}
$$

into relation (3.39) to obtain

$$
c_{23} t^{2}<\sqrt{c_{33}} \sqrt{c_{11}+c_{22}\left(t^{2}-\frac{c_{13}}{c_{23}}\right)^{2}+2\left(c_{12}+2 c_{66}\right)\left(t^{2}-\frac{c_{13}}{c_{23}}\right)} \text { for all } t>0 .
$$


It follows that

$$
\begin{aligned}
& \left(c_{22} c_{33}-c_{23}^{2}\right) t^{4}+2\left[c_{33}\left(c_{12}+2 c_{66}\right)-c_{22} c_{33} \frac{c_{13}}{c_{23}}\right] t^{2}+ \\
& +\frac{c_{33}}{c_{23}^{2}}\left[c_{11} c_{23}^{2}+c_{22} c_{13}^{2}-2\left(c_{12}+2 c_{66}\right) c_{13} c_{23}\right]>0 \text { for all } t>0
\end{aligned}
$$

The relation (3.45) is trivially satisfied when $c_{23}\left(c_{12}+2 c_{66}\right)-c_{22} c_{13} \geq 0$ so that in the subsequent analysis we will assume that

$$
c_{23}\left(c_{12}+2 c_{66}\right)-c_{22} c_{13}<0 .
$$

Since $c_{23} \in\left(0, \sqrt{c_{22} c_{33}}\right)$ we can write the relation (3.45) in the following form

$$
\begin{aligned}
& \left(\sqrt{c_{22} c_{33}-c_{23}^{2}} t^{2}-\sqrt{\frac{c_{33}}{c_{23}^{2}}\left[c_{11} c_{23}^{2}+c_{22} c_{13}^{2}-2\left(c_{12}+2 c_{66}\right) c_{13} c_{23}\right]}\right)^{2}+ \\
& +2\left\{c_{33}\left(c_{12}+2 c_{66}\right)-c_{22} c_{33} \frac{c_{13}}{c_{23}}+\right. \\
& \left.+\sqrt{c_{22} c_{33}-c_{23}^{2}} \sqrt{\frac{c_{33}}{c_{23}^{2}}\left[c_{11} c_{23}^{2}+c_{22} c_{13}^{2}-2\left(c_{12}+2 c_{66}\right) c_{13} c_{23}\right]}\right\} t^{2}>0,
\end{aligned}
$$

thus, if we set

$$
t^{2}=\frac{1}{\sqrt{c_{22} c_{33}-c_{23}^{2}}} \sqrt{\frac{c_{33}}{c_{23}^{2}}\left[c_{11} c_{23}^{2}+c_{22} c_{13}^{2}-2\left(c_{12}+2 c_{66}\right) c_{13} c_{23}\right]},
$$

we obtain the following restriction

$$
\begin{aligned}
& c_{33}\left(c_{12}+2 c_{66}\right)-c_{22} c_{33} \frac{c_{13}}{c_{23}}+ \\
& +\sqrt{c_{22} c_{33}-c_{23}^{2}} \sqrt{\frac{c_{33}}{c_{23}^{2}}\left[c_{11} c_{23}^{2}+c_{22} c_{13}^{2}-2\left(c_{12}+2 c_{66}\right) c_{13} c_{23}\right]}>0,
\end{aligned}
$$

that is,

$$
\begin{aligned}
& \sqrt{1-\frac{c_{23}^{2}}{c_{22} c_{33}}} \sqrt{\frac{c_{13}^{2}}{c_{11} c_{33}}+\frac{c_{23}^{2}}{c_{22} c_{33}}-2 \frac{c_{13}}{\sqrt{c_{11} c_{33}}} \frac{c_{23}}{\sqrt{c_{22} c_{33}}} \frac{c_{12}+2 c_{66}}{\sqrt{c_{11} c_{22}}}}> \\
& >\frac{c_{13}}{\sqrt{c_{11} c_{33}}}-\frac{c_{23}}{\sqrt{c_{22} c_{33}}} \frac{c_{12}+2 c_{66}}{\sqrt{c_{11} c_{22}}},
\end{aligned}
$$

under the assumption (3.46). Further, from the relation (3.50) we obtain

$$
\frac{\left(c_{12}+2 c_{66}\right)^{2}}{c_{11} c_{22}}+\frac{c_{13}^{2}}{c_{11} c_{33}}+\frac{c_{23}^{2}}{c_{22} c_{33}}-2 \frac{c_{13}}{\sqrt{c_{11} c_{33}}} \frac{c_{23}}{\sqrt{c_{22} c_{33}}} \frac{c_{12}+2 c_{66}}{\sqrt{c_{11} c_{22}}}-1<0,
$$


which can be solved with respect to $\frac{c_{12}+2 c_{66}}{\sqrt{c_{11} c_{22}}}$ in order to obtain

$$
\begin{aligned}
-1 & <\frac{c_{13}}{\sqrt{c_{11} c_{33}}} \frac{c_{23}}{\sqrt{c_{22} c_{33}}}-\sqrt{\left(1-\frac{c_{13}^{2}}{c_{11} c_{33}}\right)\left(1-\frac{c_{23}^{2}}{c_{22} c_{33}}\right)}<\frac{c_{12}+2 c_{66}}{\sqrt{c_{11} c_{22}}}< \\
& <\frac{c_{13}}{\sqrt{c_{11} c_{33}}} \frac{c_{23}}{\sqrt{c_{22} c_{33}}}+\sqrt{\left(1-\frac{c_{13}^{2}}{c_{11} c_{33}}\right)\left(1-\frac{c_{23}^{2}}{c_{22} c_{33}}\right)},
\end{aligned}
$$

provided that $c_{55}<\sqrt{c_{11} c_{33}}$. Thus, we have again an improvement of the estimate given by the relation (3.28) for $\frac{c_{12}+2 c_{66}}{\sqrt{c_{11} c_{22}}}$.

The case (4) can be treated in the same manner and hence we are led to

$$
\begin{aligned}
& \sqrt{1-\frac{c_{13}^{2}}{c_{11} c_{33}}} \sqrt{\frac{c_{13}^{2}}{c_{11} c_{33}}+\frac{c_{23}^{2}}{c_{22} c_{33}}-2 \frac{c_{13}}{\sqrt{c_{11} c_{33}}} \frac{c_{23}}{\sqrt{c_{22} c_{33}}} \frac{c_{12}+2 c_{66}}{\sqrt{c_{11} c_{22}}}}> \\
& >\frac{c_{23}}{\sqrt{c_{22} c_{33}}}-\frac{c_{13}}{\sqrt{c_{11} c_{33}}} \frac{c_{12}+2 c_{66}}{\sqrt{c_{11} c_{22}}},
\end{aligned}
$$

under the assumption

$$
c_{13}\left(c_{12}+2 c_{66}\right)-c_{11} c_{23}<0 \text {. }
$$

It follows that if we solve inequality (3.53) in terms of $\frac{c_{12}+2 c_{66}}{\sqrt{c_{11} c_{22}}}$, then we are led to the same expression as in (3.52), provided that $c_{44}<\sqrt{c_{22} c_{33}}$.

Concluding this subsection we note that the relation (3.20) furnishes, in the case under discussion, the sharper estimates (3.38) and (3.52) for $\frac{c_{12}+2 c_{66}}{\sqrt{c_{11} c_{22}}}$. They are more restrictive than those described by the relation (3.28) since

$$
\frac{c_{12}+2 c_{66}}{\sqrt{c_{11} c_{22}}}>\varkappa_{3}^{i}>-1
$$

where

$$
\varkappa_{3}^{i}=x y-\sqrt{\left(1-x^{2}\right)\left(1-y^{2}\right)}, \quad(x, y) \in\left(-\frac{c_{44}}{\sqrt{c_{22} c_{33}}}, 1\right) \times\left(-\frac{c_{55}}{\sqrt{c_{11} c_{33}}}, 1\right),
$$

and $x, y$ denotes respectively, $\frac{c_{23}}{\sqrt{c_{22} c_{33}}}$ and $\frac{c_{13}}{\sqrt{c_{11} c_{33}}}$, provided $c_{44}<\sqrt{c_{22} c_{33}}, c_{55}<$ $\sqrt{c_{11} c_{33}}$.

\subsubsection{Analysis of (3.21)}

We now study the relation (3.21) under the assumption (3.31). If we set $m_{2}=\gamma m_{1}$, then the relation (3.21) yields

$$
\left|-\left(c_{13}+c_{55}\right)+\left(c_{23}+c_{44}\right) \gamma^{2}\right|<c_{55}+c_{44} \gamma^{2}+\sqrt{c_{33}\left(c_{11}+c_{22} \gamma^{4}-2 c_{12} \gamma^{2}\right)} \text { for all } \gamma \in \mathbb{R} \text {. }
$$


Let us assume first that

$$
|\gamma| \leq \sqrt{\frac{c_{13}+c_{55}}{c_{23}+c_{44}}}
$$

Then, relation (3.57) becomes

$$
c_{13}+c_{55}-\left(c_{23}+c_{44}\right) \gamma^{2}<c_{55}+c_{44} \gamma^{2}+\sqrt{c_{33}\left(c_{11}+c_{22} \gamma^{4}-2 c_{12} \gamma^{2}\right)}
$$

and hence we have

$$
c_{13}-\left(c_{23}+2 c_{44}\right) \gamma^{2}<\sqrt{c_{33}\left(c_{11}+c_{22} \gamma^{4}-2 c_{12} \gamma^{2}\right)} \text { for }|\gamma| \leq \sqrt{\frac{c_{13}+c_{55}}{c_{23}+c_{44}}} .
$$

It can be easily seen that inequality (3.60) is satisfied trivially when $c_{13} \in\left(-c_{55}, 0\right]$, so we shall assume that

$$
0<c_{13}<\sqrt{c_{11} c_{33}}
$$

Further, we set

$$
\delta=\frac{1}{|\gamma|}
$$

in (3.60) to rewrite it as

$$
c_{13} \delta^{2}-\left(c_{23}+2 c_{44}\right)<\sqrt{c_{33}\left(c_{11} \delta^{4}+c_{22}-2 c_{12} \delta^{2}\right)} \text { for all } \delta \geq \sqrt{\frac{c_{23}+c_{44}}{c_{13}+c_{55}}} .
$$

Inequality (3.63) is trivially satisfied for $\sqrt{\frac{c_{23}+c_{44}}{c_{13}+c_{55}}} \leq \delta \leq \sqrt{\frac{c_{23}+2 c_{44}}{c_{13}}}$, or when $c_{12} \in$ $\left(-2 c_{66}-\sqrt{c_{11} c_{22}}, 0\right]$, so that, in what follows, we shall consider only the case when

$$
\delta>\sqrt{\frac{c_{23}+2 c_{44}}{c_{13}}} \text { and } c_{12} \in\left(0, \sqrt{c_{11} c_{22}}\right)
$$

and we set

$$
r=\sqrt{\delta^{2}-\frac{c_{23}+2 c_{44}}{c_{13}}}
$$

Inequality (3.63) yields

$$
\begin{aligned}
& \left(c_{11} c_{33}-c_{13}^{2}\right) r^{4}+2 \frac{c_{33}}{c_{13}}\left[c_{11}\left(c_{23}+2 c_{44}\right)-c_{12} c_{13}\right] r^{2}+ \\
& +\frac{c_{33}}{c_{13}^{2}}\left[c_{11}\left(c_{23}+2 c_{44}\right)^{2}+c_{22} c_{13}^{2}-2 c_{12} c_{13}\left(c_{23}+2 c_{44}\right)\right]>0 \text { for all } r>0 .
\end{aligned}
$$

Note that the relation (3.66) is trivially satisfied for $c_{11}\left(c_{23}+2 c_{44}\right)-c_{12} c_{13} \geq 0$, so that we shall discuss only the situation

$$
c_{11}\left(c_{23}+2 c_{44}\right)-c_{12} c_{13}<0 .
$$


We use the relation (3.61) and rewrite (3.66) in the form

$$
\begin{aligned}
& \left(\sqrt{c_{11} c_{33}-c_{13}^{2}} r^{2}-\frac{\sqrt{c_{33}}}{c_{13}} \sqrt{c_{11}\left(c_{23}+2 c_{44}\right)^{2}+c_{22} c_{13}^{2}-2 c_{12} c_{13}\left(c_{23}+2 c_{44}\right)}\right)^{2}+ \\
& +\frac{2}{c_{13}} c_{11} c_{33} \sqrt{c_{22} c_{33}}\left[\frac{c_{23}+2 c_{44}}{\sqrt{c_{22} c_{33}}}-\frac{c_{12}}{\sqrt{c_{11} c_{22}}} \frac{c_{13}}{\sqrt{c_{11} c_{33}}}+\right. \\
& +\sqrt{1-\frac{c_{13}^{2}}{c_{11} c_{33}}} \sqrt{\left.\frac{\left(c_{23}+2 c_{44}\right)^{2}}{c_{22} c_{33}}+\frac{c_{13}^{2}}{c_{11} c_{33}}-2 \frac{c_{12}}{\sqrt{c_{11} c_{22}}} \frac{c_{13}}{\sqrt{c_{11} c_{33}}} \frac{c_{23}+2 c_{44}}{\sqrt{c_{22} c_{33}}}\right] r^{2}>0,}
\end{aligned}
$$

for all $r \geq 0$. If we set

$$
r^{2}=\frac{\sqrt{c_{33}}}{c_{13} \sqrt{c_{11} c_{33}-c_{13}^{2}}} \sqrt{c_{11}\left(c_{23}+2 c_{44}\right)^{2}+c_{22} c_{13}^{2}-2 c_{12} c_{13}\left(c_{23}+2 c_{44}\right)},
$$

then (3.68) gives

$$
\begin{aligned}
& \frac{c_{23}+2 c_{44}}{\sqrt{c_{22} c_{33}}}-\frac{c_{12}}{\sqrt{c_{11} c_{22}}} \frac{c_{13}}{\sqrt{c_{11} c_{33}}}+ \\
& \quad+\sqrt{1-\frac{c_{13}^{2}}{c_{11} c_{33}}} \sqrt{\frac{\left(c_{23}+2 c_{44}\right)^{2}}{c_{22} c_{33}}+\frac{c_{13}^{2}}{c_{11} c_{33}}-2 \frac{c_{12}}{\sqrt{c_{11} c_{22}}} \frac{c_{13}}{\sqrt{c_{11} c_{33}}} \frac{c_{23}+2 c_{44}}{\sqrt{c_{22} c_{33}}}}>0
\end{aligned}
$$

and hence, by solving with respect to $\frac{c_{23}+2 c_{44}}{\sqrt{c_{22} c_{33}}}$, we obtain

$$
\begin{aligned}
-1 & <\frac{c_{13}}{\sqrt{c_{11} c_{33}}} \frac{c_{12}}{\sqrt{c_{11} c_{22}}}-\sqrt{\left(1-\frac{c_{13}^{2}}{c_{11} c_{33}}\right)\left(1-\frac{c_{12}^{2}}{c_{11} c_{22}}\right)}<\frac{c_{23}+2 c_{44}}{\sqrt{c_{22} c_{33}}}< \\
& <\frac{c_{13}}{\sqrt{c_{11} c_{33}}} \frac{c_{12}}{\sqrt{c_{11} c_{22}}}+\sqrt{\left(1-\frac{c_{13}^{2}}{c_{11} c_{33}}\right)\left(1-\frac{c_{12}^{2}}{c_{11} c_{22}}\right)}<1 .
\end{aligned}
$$

In a similar manner we can treat the case when

$$
|\gamma|>\sqrt{\frac{c_{13}+c_{55}}{c_{23}+c_{44}}}
$$

in order to show that inequality (3.57) implies

$$
\begin{gathered}
-1<\frac{c_{23}}{\sqrt{c_{22} c_{33}}} \frac{c_{12}}{\sqrt{c_{11} c_{22}}}-\sqrt{\left(1-\frac{c_{23}^{2}}{c_{22} c_{33}}\right)\left(1-\frac{c_{12}^{2}}{c_{11} c_{22}}\right)}<\frac{c_{13}+2 c_{55}}{\sqrt{c_{11} c_{33}}}< \\
<\frac{c_{23}}{\sqrt{c_{22} c_{33}}} \frac{c_{12}}{\sqrt{c_{11} c_{22}}}+\sqrt{\left(1-\frac{c_{23}^{2}}{c_{22} c_{33}}\right)\left(1-\frac{c_{12}^{2}}{c_{11} c_{22}}\right)}<1 .
\end{gathered}
$$


3.1.3 Short Study of the Case $c_{13}+c_{55}<0, c_{23}+c_{44}<0$

In this case we have

$$
-2 c_{55}-\sqrt{c_{11} c_{33}}<c_{13}<-c_{55}, \quad-2 c_{44}-\sqrt{c_{22} c_{33}}<c_{23}<-c_{44}
$$

and therefore (3.20) becomes

$$
-\left(c_{13}+2 c_{55}\right) m_{1}^{2}-\left(c_{23}+2 c_{44}\right) m_{2}^{2}<\sqrt{c_{33}} \sqrt{c_{11} m_{1}^{4}+c_{22} m_{2}^{4}+2\left(c_{12}+2 c_{66}\right) m_{1}^{2} m_{2}^{2}},
$$

for all $\left(m_{1}, m_{2}\right) \neq \mathbf{0}$.

If we set

$$
\bar{c}_{13}=-\left(c_{13}+2 c_{55}\right), \quad \bar{c}_{23}=-\left(c_{23}+2 c_{44}\right),
$$

in (3.74) we have

$$
-c_{55}<\bar{c}_{13}<\sqrt{c_{11} c_{33}}, \quad-c_{44}<\bar{c}_{23}<\sqrt{c_{22} c_{33}}
$$

and (3.75) becomes

$$
\bar{c}_{13} m_{1}^{2}+\bar{c}_{23} m_{2}^{2}<\sqrt{c_{33}} \sqrt{c_{11} m_{1}^{4}+c_{22} m_{2}^{4}+2\left(c_{12}+2 c_{66}\right) m_{1}^{2} m_{2}^{2}} .
$$

It can be easily seen that (3.77) and (3.78) are similar to (3.31) and (3.32), respectively, and hence we can use the previous analysis in order to obtain the following improved estimates for $\frac{c_{12}+2 c_{66}}{\sqrt{c_{11} c_{22}}}$ as

$$
\begin{aligned}
& \frac{c_{12}+2 c_{66}}{\sqrt{c_{11} c_{22}}}>\frac{\bar{c}_{13}}{\sqrt{c_{11} c_{33}}} \frac{\bar{c}_{23}}{\sqrt{c_{22} c_{33}}}-\sqrt{\left(1-\frac{\bar{c}_{13}^{2}}{c_{11} c_{33}}\right)\left(1-\frac{\bar{c}_{23}^{2}}{c_{22} c_{33}}\right)}>-1, \\
&-1<\frac{\bar{c}_{13}}{\sqrt{c_{11} c_{33}}} \frac{\bar{c}_{23}}{\sqrt{c_{22} c_{33}}}-\sqrt{\left(1-\frac{\bar{c}_{13}^{2}}{c_{11} c_{33}}\right)\left(1-\frac{\bar{c}_{23}^{2}}{c_{22} c_{33}}\right)}<\frac{c_{12}+2 c_{66}}{\sqrt{c_{11} c_{22}}}< \\
&<\frac{\bar{c}_{13}}{\sqrt{c_{11} c_{33}}} \frac{\bar{c}_{23}}{\sqrt{c_{22} c_{33}}}+\sqrt{\left(1-\frac{\bar{c}_{13}^{2}}{c_{11} c_{33}}\right)\left(1-\frac{\bar{c}_{23}^{2}}{c_{22} c_{33}}\right)}
\end{aligned}
$$

under the assumptions $c_{44}<\sqrt{c_{22} c_{33}}, c_{55}<\sqrt{c_{11} c_{33}}$ and

$$
c_{22}\left(c_{13}+2 c_{55}\right)-\left(c_{12}+2 c_{66}\right)\left(c_{23}+2 c_{44}\right)<0
$$

or

$$
c_{11}\left(c_{23}+2 c_{44}\right)-\left(c_{12}+2 c_{66}\right)\left(c_{13}+2 c_{55}\right)<0 .
$$

Thus, in the present case the relation (3.20) furnishes the estimates described by (3.79) and (3.80).

Concluding the analysis of this case we can assert that the relation (3.20) furnishes the following lower bound

$$
\frac{c_{12}+2 c_{66}}{\sqrt{c_{11} c_{22}}}>\varkappa_{3}^{i}>-1
$$


where now

$$
\varkappa_{3}^{i}=x y-\sqrt{\left(1-x^{2}\right)\left(1-y^{2}\right)}, \quad(x, y) \in\left(-1,-\frac{c_{44}}{\sqrt{c_{22} c_{33}}}\right) \times\left(-1,-\frac{c_{55}}{\sqrt{c_{11} c_{33}}}\right)
$$

and $x$ is $\frac{\bar{c}_{23}}{\sqrt{c_{22} c_{33}}}$ and $y$ is $\frac{\bar{c}_{13}}{\sqrt{c_{11} c_{33}}}$, provided $c_{44}<\sqrt{c_{22} c_{33}}, c_{55}<\sqrt{c_{11} c_{33}}$.

Concerning (3.21), we have to note that the analysis follows the same procedure as in the case $c_{13}+c_{55}>0, c_{23}+c_{44}>0$ leading to conclusions (3.71) and (3.73).

3.2 The Case $\left(c_{13}+c_{55}\right) \cdot\left(c_{23}+c_{44}\right)<0$

For convenience we will first suppose that

$$
c_{13}+c_{55}<0, \quad c_{23}+c_{44}>0 .
$$

In view of the relation (3.30), we have to study the following situation

$$
-2 c_{55}-\sqrt{c_{11} c_{33}}<c_{13}<-c_{55}, \quad-c_{44}<c_{23}<\sqrt{c_{22} c_{33}} .
$$

We note that the analysis of (3.20) and (3.21) in this case has some symmetry with respect to the case $\left(c_{13}+c_{55}\right) \cdot\left(c_{23}+c_{44}\right)>0$. Thus, we start our study with the relation (3.21).

\subsubsection{Analysis of (3.21)}

The relation (3.21) gives

$$
-\left(c_{13}+2 c_{55}\right) m_{1}^{2}+c_{23} m_{2}^{2}<\sqrt{c_{33}} \sqrt{c_{11} m_{1}^{4}+c_{22} m_{2}^{4}-2 c_{12} m_{1}^{2} m_{2}^{2}} \text { for all }\left(m_{1}, m_{2}\right) \neq \mathbf{0}
$$

Using a notation previously introduced in (3.76), that is $\bar{c}_{13}=-\left(c_{13}+2 c_{55}\right)$, relation (3.86) becomes

$$
-c_{55}<\bar{c}_{13}<\sqrt{c_{11} c_{33}}, \quad-c_{44}<c_{23}<\sqrt{c_{22} c_{33}},
$$

while the relation $(3.87)$ reads

$$
\bar{c}_{13} m_{1}^{2}+c_{23} m_{2}^{2}<\sqrt{c_{33}} \sqrt{c_{11} m_{1}^{4}+c_{22} m_{2}^{4}-2 c_{12} m_{1}^{2} m_{2}^{2}} \text { for all }\left(m_{1}, m_{2}\right) \neq \mathbf{0} .
$$

Thus, we have to discuss the consequences of the relation (3.89) in the following four cases:

(a) $\bar{c}_{13} \in\left(-c_{55}, 0\right]$ and $c_{23} \in\left(-c_{44}, 0\right]$;

(b) $\bar{c}_{13} \in\left(0, \sqrt{c_{11} c_{33}}\right)$ and $c_{23} \in\left(0, \sqrt{c_{22} c_{33}}\right)$;

(c) $\bar{c}_{13} \in\left(-c_{55}, 0\right]$ and $c_{23} \in\left(0, \sqrt{c_{22} c_{33}}\right)$;

(d) $\bar{c}_{13} \in\left(0, \sqrt{c_{11} c_{33}}\right)$ and $c_{23} \in\left(-c_{44}, 0\right]$.

In case (a) inequality (3.89) is trivially satisfied. In order to discuss the case (b) we recall that (3.13) gives

$$
-\sqrt{c_{11} c_{22}}<-c_{12}<2 c_{66}+\sqrt{c_{11} c_{22}} .
$$


For

$$
\sqrt{c_{11} c_{22}} \leq-c_{12}<2 c_{66}+\sqrt{c_{11} c_{22}},
$$

inequality (3.89) is trivially satisfied because we have

$$
\begin{aligned}
\bar{c}_{13} m_{1}^{2}+c_{23} m_{2}^{2} & =\sqrt{\bar{c}_{13}^{2} m_{1}^{4}+c_{23}^{2} m_{2}^{4}+2 \bar{c}_{13} c_{23} m_{1}^{2} m_{2}^{2}}< \\
& <\sqrt{c_{11} c_{33} m_{1}^{4}+c_{22} c_{33} m_{2}^{4}+2 c_{33} \sqrt{c_{11} c_{22}} m_{1}^{2} m_{2}^{2}} \leq \\
& \leq \sqrt{c_{11} c_{33} m_{1}^{4}+c_{22} c_{33} m_{2}^{4}-2 c_{33} c_{12} m_{1}^{2} m_{2}^{2}} .
\end{aligned}
$$

So in what follows we shall study the case (b) under the assumption

$$
-\sqrt{c_{11} c_{22}}<-c_{12}<\sqrt{c_{11} c_{22}} \text {. }
$$

Then inequality (3.89) gives

$$
\begin{aligned}
& \left(\sqrt{c_{11} c_{33}-\bar{c}_{13}^{2}} m_{1}^{2}-\sqrt{c_{22} c_{33}-c_{23}^{2}} m_{2}^{2}\right)^{2}+ \\
& \quad+2\left[\sqrt{c_{11} c_{33}-\bar{c}_{13}^{2}} \sqrt{c_{22} c_{33}-c_{23}^{2}}-c_{33} c_{12}-\bar{c}_{13} c_{23}\right] m_{1}^{2} m_{2}^{2}>0
\end{aligned}
$$

and hence we deduce

$$
\sqrt{c_{11} c_{33}-\bar{c}_{13}^{2}} \sqrt{c_{22} c_{33}-c_{23}^{2}}-c_{33} c_{12}-\bar{c}_{13} c_{23}>0 .
$$

Thus, (3.89) yields

$$
\frac{c_{12}}{\sqrt{c_{11} c_{22}}}<\frac{c_{13}+2 c_{55}}{\sqrt{c_{11} c_{33}}} \frac{c_{23}}{\sqrt{c_{22} c_{33}}}+\sqrt{\left[1-\frac{\left(c_{13}+2 c_{55}\right)^{2}}{c_{11} c_{33}}\right]\left(1-\frac{c_{23}^{2}}{c_{22} c_{33}}\right)}<1 .
$$

Let us now consider the case (c). We set $m_{2}=\sigma m_{1}, \sigma \in \mathbb{R}$, and rewrite (3.89) as

$$
\bar{c}_{13}+c_{23} \sigma^{2}<\sqrt{c_{11} c_{33}+c_{22} c_{33} \sigma^{4}-2 c_{33} c_{12} \sigma^{2}} \text { for all } \sigma \in \mathbb{R} .
$$

We recall now (3.13), that is $-2 c_{66}-\sqrt{c_{11} c_{22}}<c_{12}<\sqrt{c_{11} c_{22}}$. For $-2 c_{66}-\sqrt{c_{11} c_{22}}<$ $c_{12} \leq 0$ it is easy to see that the relation (3.97) is trivially satisfied because we have

$$
\bar{c}_{13}+c_{23} \sigma^{2}<\sqrt{c_{22} c_{33} \sigma^{4}}<\sqrt{c_{11} c_{33}+c_{22} c_{33} \sigma^{4}-2 c_{33} c_{12} \sigma^{2}} .
$$

So we shall discuss the case (c) when

$$
0<c_{12}<\sqrt{c_{11} c_{22}} \text {. }
$$

We first observe that the relation (3.97) is trivially satisfied for all $\sigma \in \mathbb{R}$, with $\sigma^{2} \leq$ $-\frac{\bar{c}_{13}}{c_{23}}$. Further, we assume that $\sigma^{2}>-\frac{\bar{c}_{13}}{c_{23}}$ and we introduce $\sigma=\sqrt{s^{2}-\frac{\bar{c}_{13}}{c_{23}}}, s \in \mathbb{R}$ in (3.97) and obtain

$$
\left(c_{22} c_{33}-c_{23}^{2}\right) s^{4}-2\left(c_{33} c_{12}+c_{22} c_{33} \frac{\bar{c}_{13}}{c_{23}}\right) s^{2}+\frac{c_{33}}{c_{23}^{2}}\left[c_{11} c_{23}^{2}+c_{22} \bar{c}_{13}^{2}+2 c_{12} c_{23} \bar{c}_{13}\right]>0,
$$


for all $s \in \mathbb{R}$. This inequality is trivially satisfied when $c_{12} c_{23}-c_{22}\left(c_{13}+2 c_{55}\right) \leq 0$, so that, in our next subsequent analysis we shall assume

$$
c_{12} c_{23}-c_{22}\left(c_{13}+2 c_{55}\right)>0 \text {. }
$$

In this case from (3.100), we deduce that

$$
\begin{aligned}
& -\frac{\bar{c}_{13}}{\sqrt{c_{11} c_{33}}}-\frac{c_{12}}{\sqrt{c_{11} c_{22}}} \frac{c_{23}}{\sqrt{c_{22} c_{33}}}+ \\
& +\sqrt{1-\frac{c_{23}^{2}}{c_{22} c_{33}}} \sqrt{\frac{c_{23}^{2}}{c_{22} c_{33}}+\frac{\bar{c}_{13}^{2}}{c_{11} c_{33}}+2 \frac{c_{12}}{\sqrt{c_{11} c_{22}}} \frac{c_{23}}{\sqrt{c_{22} c_{33}}} \frac{\bar{c}_{13}}{\sqrt{c_{11} c_{33}}}}>0 .
\end{aligned}
$$

Solving this inequality with respect to $\frac{c_{12}}{\sqrt{c_{11} c_{22}}}$ we obtain the following estimate

$$
\begin{gathered}
\frac{c_{23}}{\sqrt{c_{22} c_{33}}} \frac{c_{13}+2 c_{55}}{\sqrt{c_{11} c_{33}}}-\sqrt{\left(1-\frac{c_{23}^{2}}{c_{22} c_{33}}\right)\left[1-\frac{\left(c_{13}+2 c_{55}\right)^{2}}{c_{11} c_{33}}\right]}<\frac{c_{12}}{\sqrt{c_{11} c_{22}}}< \\
<\frac{c_{23}}{\sqrt{c_{22} c_{33}}} \frac{c_{13}+2 c_{55}}{\sqrt{c_{11} c_{33}}}+\sqrt{\left(1-\frac{c_{23}^{2}}{c_{22} c_{33}}\right)\left[1-\frac{\left(c_{13}+2 c_{55}\right)^{2}}{c_{11} c_{33}}\right]}<1,
\end{gathered}
$$

provided $c_{55}<\sqrt{c_{11} c_{33}}$.

The case (d) can be treated in a similar manner in order to furnish the same estimate as (3.102) under the assumption that

$$
c_{12}\left(c_{13}+2 c_{55}\right)-c_{11} c_{23}<0 .
$$

Finally, let us consider the case $c_{13}+c_{55}>0, c_{23}+c_{44}<0$. Then a straightforward computation leads to the following estimates

$$
\begin{gathered}
\frac{c_{12}}{\sqrt{c_{11} c_{22}}}<\frac{c_{13}}{\sqrt{c_{11} c_{33}}} \frac{c_{23}+2 c_{44}}{\sqrt{c_{22} c_{33}}}+\sqrt{\left(1-\frac{c_{13}^{2}}{c_{11} c_{33}}\right)\left[1-\frac{\left(c_{23}+2 c_{44}\right)^{2}}{c_{22} c_{33}}\right]}<1,(3.10 \\
\frac{c_{23}+2 c_{44}}{\sqrt{c_{22} c_{33}}} \frac{c_{13}}{\sqrt{c_{11} c_{33}}}-\sqrt{\left(1-\frac{c_{13}^{2}}{c_{11} c_{33}}\right)\left[1-\frac{\left(c_{23}+2 c_{44}\right)^{2}}{c_{22} c_{33}}\right]}<\frac{c_{12}}{\sqrt{c_{11} c_{22}}}< \\
<\frac{c_{23}+2 c_{44}}{\sqrt{c_{22} c_{33}}} \frac{c_{13}}{\sqrt{c_{11} c_{33}}}+\sqrt{\left(1-\frac{c_{13}^{2}}{c_{11} c_{33}}\right)\left[1-\frac{\left(c_{23}+2 c_{44}\right)^{2}}{c_{22} c_{33}}\right]}<1
\end{gathered}
$$

provided $c_{44}<\sqrt{c_{22} c_{33}}$.

In the present context, (3.21) imposes the estimates (3.96), (3.103), (3.105) and (3.106) instead of those described by (3.28). Thus, we have

$$
\frac{c_{12}}{\sqrt{c_{11} c_{22}}}<\varkappa_{3}^{s}<1
$$


where

$$
\varkappa_{3}^{s}=x y+\sqrt{\left(1-x^{2}\right)\left(1-y^{2}\right)}, \quad(x, y) \in(-1,1) \times(-1,1),
$$

and $x \in\left\{\frac{c_{23}}{\sqrt{c_{22} c_{33}}}, \frac{c_{23}+2 c_{44}}{\sqrt{c_{22} c_{33}}}\right\}$ and $y \in\left\{\frac{c_{13}}{\sqrt{c_{11} c_{33}}}, \frac{c_{13}+2 c_{55}}{\sqrt{c_{11} c_{33}}}\right\}$ and $c_{44}<\sqrt{c_{22} c_{33}}, c_{55}<\sqrt{c_{11} c_{33}}$.

\subsubsection{Analysis of (3.20)}

In what follows we shall assume that the relation (3.85) holds and proceed to study (3.20). We set $m_{2}=\tau m_{1}, \tau \in \mathbb{R}$, in (3.20) and obtain

$$
\left|c_{13}+c_{55}+\left(c_{23}+c_{44}\right) \tau^{2}\right|<c_{55}+c_{44} \tau^{2}+\sqrt{c_{11} c_{33}+c_{22} c_{33} \tau^{4}+2 c_{33}\left(c_{12}+2 c_{66}\right) \tau^{2}},
$$

for all $\tau \in \mathbb{R}$.

If we assume

$$
|\tau|>\sqrt{-\frac{c_{13}+c_{55}}{c_{23}+c_{44}}},
$$

then (3.109) gives

$$
c_{13}+c_{23} \tau^{2}<\sqrt{c_{11} c_{33}+c_{22} c_{33} \tau^{4}+2 c_{33}\left(c_{12}+2 c_{66}\right) \tau^{2}} .
$$

We note that this inequality is obvious satisfied if $c_{23} \in\left(-c_{44}, 0\right]$ or $c_{12} \in\left[0,2 c_{66}+\right.$ $\sqrt{c_{11} c_{22}}$ ), so that in what follows we shall assume

$$
c_{23} \in\left(0, \sqrt{c_{22} c_{33}}\right), \quad c_{12} \in\left(-\sqrt{c_{11} c_{22}}, 0\right) .
$$

The method used to study (3.73) provides here

$$
\begin{aligned}
& \sqrt{1-\frac{c_{23}^{2}}{c_{22} c_{33}}} \sqrt{\frac{c_{23}^{2}}{c_{22} c_{33}}+\frac{c_{13}^{2}}{c_{11} c_{33}}-2 \frac{c_{12}+2 c_{66}}{\sqrt{c_{11} c_{22}}} \frac{c_{13}}{\sqrt{c_{11} c_{33}}} \frac{c_{23}}{\sqrt{c_{22} c_{33}}}}> \\
& >\frac{c_{13}}{\sqrt{c_{11} c_{33}}}-\frac{c_{23}}{\sqrt{c_{22} c_{33}}} \frac{c_{12}+2 c_{66}}{\sqrt{c_{11} c_{22}}},
\end{aligned}
$$

which when solved with respect to $\frac{c_{12}+2 c_{66}}{\sqrt{c_{11} c_{22}}}$, gives

$$
\begin{aligned}
& \frac{c_{13}}{\sqrt{c_{11} c_{33}}} \frac{c_{23}}{\sqrt{c_{22} c_{33}}}-\sqrt{\left(1-\frac{c_{13}^{2}}{c_{11} c_{33}}\right)\left(1-\frac{c_{23}^{2}}{c_{22} c_{33}}\right)}<\frac{c_{12}+2 c_{66}}{\sqrt{c_{11} c_{22}}}< \\
& <\frac{c_{13}}{\sqrt{c_{11} c_{33}}} \frac{c_{23}}{\sqrt{c_{22} c_{33}}}+\sqrt{\left(1-\frac{c_{13}^{2}}{c_{11} c_{33}}\right)\left(1-\frac{c_{23}^{2}}{c_{22} c_{33}}\right)} .
\end{aligned}
$$

If we consider the case

$$
0<\tau \leq \sqrt{-\frac{c_{13}+c_{55}}{c_{23}+c_{44}}}
$$


inequality (3.109) gives

$-\left(c_{13}+2 c_{55}\right)-\left(c_{23}+2 c_{44}\right) \tau^{2}<\sqrt{c_{11} c_{33}+c_{22} c_{33} \tau^{4}+2 c_{33}\left(c_{12}+2 c_{66}\right) \tau^{2}}$, for all $\tau \in \mathbb{R}$.

Using for (3.116) the same procedure as the one used to study (3.59) leads to

$$
\begin{aligned}
& \sqrt{1-\frac{\left(c_{23}+2 c_{44}\right)^{2}}{c_{22} c_{33}}} \sqrt{\frac{\left(c_{23}+2 c_{44}\right)^{2}}{c_{22} c_{33}}+\frac{\left(c_{13}+2 c_{55}\right)^{2}}{c_{11} c_{33}}-2 \frac{c_{12}+2 c_{66}}{\sqrt{c_{11} c_{22}}} \frac{c_{13}+2 c_{55}}{\sqrt{c_{11} c_{33}}} \frac{c_{23}+2 c_{44}}{\sqrt{c_{22} c_{33}}}>} \\
& >\frac{c_{13}+2 c_{55}}{\sqrt{c_{11} c_{33}}}-\frac{c_{23}+2 c_{44}}{\sqrt{c_{22} c_{33}}} \frac{c_{12}+2 c_{66}}{\sqrt{c_{11} c_{22}}},
\end{aligned}
$$

which further gives

$$
\begin{gathered}
\frac{c_{13}+2 c_{55}}{\sqrt{c_{11} c_{33}}} \frac{c_{23}+2 c_{44}}{\sqrt{c_{22} c_{33}}}-\sqrt{\left[1-\frac{\left(c_{13}+2 c_{55}\right)^{2}}{c_{11} c_{33}}\right]\left[1-\frac{\left(c_{23}+2 c_{44}\right)^{2}}{c_{22} c_{33}}\right]}<\frac{c_{12}+2 c_{66}}{\sqrt{c_{11} c_{22}}}< \\
<\frac{c_{13}+2 c_{55}}{\sqrt{c_{11} c_{33}}} \frac{c_{23}+2 c_{44}}{\sqrt{c_{22} c_{33}}}-\sqrt{\left[1-\frac{\left(c_{13}+2 c_{55}\right)^{2}}{c_{11} c_{33}}\right]\left[1-\frac{\left(c_{23}+2 c_{44}\right)^{2}}{c_{22} c_{33}}\right]} .
\end{gathered}
$$

Combining the results obtained in the last two cases we see that (3.20) gives an upper bound for $\frac{c_{12}}{\sqrt{c_{11} c_{22}}}$ as

$$
\frac{c_{12}}{\sqrt{c_{11} c_{22}}}<\varkappa_{3}^{s}<1
$$

where

$$
\varkappa_{3}^{s}=x y+\sqrt{\left(1-x^{2}\right)\left(1-y^{2}\right)}, \quad(x, y) \in(-1,1) \times(-1,1) .
$$

\subsection{Overview}

Collecting together the previously obtained results we conclude that the strong ellipticity condition (2.3) is equivalent to the set of restrictions described by the relations (3.4), (3.9) and

$$
-2 c_{66}+\varkappa_{3}^{i} \sqrt{c_{11} c_{22}}<c_{12}<\varkappa_{3}^{s} \sqrt{c_{11} c_{22}}
$$

where $\varkappa_{3}^{i}<\varkappa_{3}^{s}$ are the solutions of the equation in the unknown $z$

$$
x^{2}+y^{2}+z^{2}-2 x y z-1=0, \quad(x, y, z) \in(-1,1) \times(-1,1) \times(-1,1) .
$$

Taking into account the symmetry of the problem, we can deduce that

$$
-2 c_{44}+\varkappa_{1}^{i} \sqrt{c_{22} c_{33}}<c_{23}<\varkappa_{1}^{s} \sqrt{c_{22} c_{33}}
$$


where $\varkappa_{1}^{i}<\varkappa_{1}^{s}$ are the solutions in $x$ of (3.122). The same argument gives further

$$
-2 c_{55}+\varkappa_{2}^{i} \sqrt{c_{11} c_{33}}<c_{13}<\varkappa_{2}^{s} \sqrt{c_{11} c_{33}},
$$

where $\varkappa_{2}^{i}<\varkappa_{2}^{s}$ are the solutions in $y$ of the equation (3.122), and this completes the proof.

\section{Consequences of Theorem 1}

\subsection{Strong Ellipticity for Transversely Isotropic Materials}

A direct consequence of the above analysis gives the proof of the Theorem 2 . Consider the crystal class of the hexagonal division. Then elasticities are restricted by either the group $\mathcal{C}_{10}$ generated by $\mathbf{R}_{\mathbf{e}_{3}}^{\pi / 3}$, or $\mathcal{C}_{11}$ generated by $\mathbf{R}_{\mathbf{e}_{3}}^{\pi / 3}$ and $\mathbf{R}_{\mathbf{e}_{2}}^{\pi}$. As noted in the Introduction, the restrictions imposed on the tensor of elasticity are identical with those imposed by transverse isotropy $\mathcal{C}_{12}$ generated by $\mathbf{R}_{\mathbf{e}_{3}}^{\varphi}, 0<\varphi<2 \pi$. In this case the relation (3.17) becomes

$$
\begin{gathered}
\left|c_{13}+c_{55}\right|\left|n_{1} m_{1}+n_{2} m_{2}\right|<c_{55} \sqrt{\left(m_{1}^{2}+m_{2}^{2}\right)\left(n_{1}^{2}+n_{2}^{2}\right)}+ \\
+\sqrt{c_{33}} \sqrt{c_{11}\left(n_{1} m_{1}+n_{2} m_{2}\right)^{2}+c_{66}\left(n_{1} m_{2}-n_{2} m_{1}\right)^{2}} .
\end{gathered}
$$

It can be easily shown that (4.1) is a direct consequence of (3.9) and (3.26). Thus, (3.4), (3.9), (3.13), (3.26) and (3.27) show that the strong ellipticity for a transversely isotropic elastic material is equivalent with

$$
c_{11}>0, \quad c_{33}>0, \quad c_{55}>0, \quad c_{11}>c_{12}, \quad\left|c_{13}+c_{55}\right|<c_{55}+\sqrt{c_{11} c_{33}},
$$

which is exactly (2.9). This completes the proof of Theorem 2 and recover the previously results of Payton [6], Padovani [7], Merodio and Ogden [8] and Chiriţă [9].

\subsection{Strong Ellipticity for 4 Classes in the Tetragonal System}

Among the seven crystal classes in the tetragonal system, for those listed at item (v) in the Introduction (four classes) restrictions on elasticities are imposed by the symmetry group $\mathcal{C}_{5}$ generated by $\mathbf{R}_{\mathbf{e}_{3}}^{\pi / 2}, \mathbf{R}_{\mathbf{e}_{1}}^{\pi}$. The number of elasticities is reduced to six and an explicit form of the elasticity tensor is obtained collecting together (2.1), (2.2) and (2.10).

In this case (3.17) becomes

$$
\begin{aligned}
\mid c_{13} & +c_{55}|| n_{1} m_{1}+n_{2} m_{2} \mid<c_{55} \sqrt{\left(m_{1}^{2}+m_{2}^{2}\right)\left(n_{1}^{2}+n_{2}^{2}\right)}+ \\
& +\sqrt{c_{33}} \sqrt{c_{11}\left(n_{1} m_{1}+n_{2} m_{2}\right)^{2}+c_{66}\left(n_{1} m_{2}-n_{2} m_{1}\right)^{2}+2\left(c_{12}-c_{11}+2 c_{66}\right) n_{1} m_{1} n_{2} m_{2}},
\end{aligned}
$$


(3.20) reads

$$
\left|c_{13}+c_{55}\right|<c_{55}+\sqrt{c_{33}} \sqrt{c_{11}+2\left(c_{12}-c_{11}+2 c_{66}\right) \frac{m_{1}^{2} m_{2}^{2}}{\left(m_{1}^{2}+m_{2}^{2}\right)^{2}}}
$$

and (3.21) becomes

$$
\left|\left(c_{13}+c_{55}\right)\left(m_{1}^{2}-m_{2}^{2}\right)\right|<c_{55}\left(m_{1}^{2}+m_{2}^{2}\right)+\sqrt{c_{33}} \sqrt{c_{11}\left(m_{1}^{4}+m_{2}^{4}\right)-2 c_{12} m_{1}^{2} m_{2}^{2}} .
$$

In view of the relation (3.26) and by using the inequality $c_{11}>c_{12}$, we have

$$
\begin{aligned}
& \left|\left(c_{13}+c_{55}\right)\left(m_{1}^{2}-m_{2}^{2}\right)\right|<\left(\sqrt{c_{11} c_{33}}+c_{55}\right)\left|m_{1}^{2}-m_{2}^{2}\right| \leq \sqrt{c_{11} c_{33}}\left|m_{1}^{2}-m_{2}^{2}\right|+c_{55}\left(m_{1}^{2}+m_{2}^{2}\right) \leq \\
& \leq \sqrt{c_{11} c_{33}\left(m_{1}^{2}-m_{2}^{2}\right)^{2}+2 c_{33}\left(c_{11}-c_{12}\right) m_{1}^{2} m_{2}^{2}}+c_{55}\left(m_{1}^{2}+m_{2}^{2}\right),
\end{aligned}
$$

so that (4.5) is trivially satisfied. It follows that the strong ellipticity condition (2.11) imposes restrictions upon the elastic coefficients only by means of the relation (4.4).

As a consequence, the strong ellipticity condition (2.11) is equivalent with the following conditions

$$
\begin{aligned}
c_{11} & >0, \quad c_{33}>0, \quad c_{55}>0, \quad c_{66}>0, \\
& -2 c_{66}+\varkappa_{3}^{i} c_{11}<c_{12}<\varkappa_{3}^{s} c_{11}, \\
& -2 c_{55}+\varkappa_{2}^{i} \sqrt{c_{11} c_{33}}<c_{13}<\varkappa_{2}^{s} \sqrt{c_{11} c_{33}},
\end{aligned}
$$

where

$$
\begin{aligned}
& \varkappa_{3}^{i}=2 x^{2}-1, \quad \varkappa_{3}^{s}=1, \text { for } x \in(-1,1), \\
& \varkappa_{2}^{i}=-\sqrt{\frac{1+z}{2}}, \quad \varkappa_{2}^{s}=\sqrt{\frac{1+z}{2}}, \text { for } z \in(-1,1),
\end{aligned}
$$

and $x=\frac{c_{13}}{\sqrt{c_{11} c_{33}}}$ or $x=\frac{c_{13}+2 c_{55}}{\sqrt{c_{11} c_{33}}}$ while $z=\frac{c_{12}}{\sqrt{c_{11} c_{22}}}$ or $z=\frac{c_{12}+2 c_{66}}{\sqrt{c_{11} c_{22}}}$. Thus the proof of Theorem 3 is complete.

Inequality (4.4) shows that the term $c_{12}-c_{11}+2 c_{66}$ is responsible for the restrictions described by the relations (4.8) and (4.9). In fact, for $c_{12}-c_{11}+2 c_{66}=0$, i.e., for a transversely isotropic elastic material, the relation (4.4) is a consequence of (3.28) and therefore it imposes no additional restriction other than that already established up to that point. In this case we have $\frac{c_{12}+2 c_{66}}{\sqrt{c_{11} c_{22}}}=1$ and hence $\varkappa_{3}^{i}=1$, that is $z=1$ and (4.11) gives $\varkappa_{2}^{i}=-1$ and $\varkappa_{2}^{s}=1$. Thus, the relation (4.9) reduces to (4.2).

Finally we note that the cubic system can by analyzed by the same method as for transverse isotropy and so we have the Theorem 4.

\section{Concluding Remarks}

Collecting together, the results of this paper give a complete characterization for strong ellipticity in terms of elastic coefficients for exactly 16 crystal classes (among 32). The key point is the proof of these conditions in the rhombic case and more symmetric situations are dealt with as particular cases. 
We have to note that, at a first glance, imposing positivity of the acoustic tensor seems to be a more natural way to address this problem as long as this condition already eliminates one of the two vectors $\mathbf{m}$ or $\mathbf{n}$. On this line, the Sylvester criterium provides a straightforward way toward conditions of positivity and one can obtain easily both (2.4) and three inequalities similar to (2.5) but where $\varkappa_{j}^{s}=\varkappa_{j}^{i}=1$ which is weaker than (2.5). The remaining difficulty is the sixth degree homogeneous polynomial expression of the determinant of the acoustic tensor, which as proved above, gives a sharper estimate than the second-order minors of the acoustic tensor. Obviously, the determinant of the acoustic tensor is bi-cubic so that one may expect some tractable restrictions from the study of conditions imposed on the coefficients of the third order polynomial in order to insure positivity on $\mathbb{R}^{+}$. When explicitly written, these conditions provide a 12-degree polynomial in two variables and despite our efforts we could not recover our (known) result on this way.

A careful inspection of the proof shows that one of the key points is the equivalence between (3.18)-(3.19) and (3.20)-(3.21) which in itself reduce the number of independent variables. Also we note that the intrinsic symmetry of the problem in the rhombic case leads to a restriction that inherits some symmetry of the problem, and the restrictions imposed by strong ellipticity lead to the simple algebraic surface $x^{2}+y^{2}+z^{2}-2 x y z-1=0$.

A natural question is the way to extend to the other crystal classes this result, or, at least, to adapt it in order to cover other anisotropic situations, a problem that will be addressed in a future work.

Finally, we have to point out that such characterization proved to be useful in discussing the uniqueness and wave propagation problems [1], loss of ellipticity in the context of nonlinear elasticity of fibre-reinforced materials [2] and in the study of spatial behaviour of the constrained anisotropic cylinder [3]. Particularly, we emphasize that our results can be useful in the study of the properties of the auxetic materials, that is materials with extreme and unusual physical properties. Among them, one has polymeric foams with low density, Evans [10], having a high capacity of absorbtion energy and high resistance to flexural deformations, Gibson and Ashby [11] thus leading to a significant loss of acoustic wave propagation, Scarpa and Tomlinson [12].

Acknowledgements The authors are very grateful to the reviewers for their suggestions and observations which led to an essential improvement of the paper. The work of S.C. was supported by grant CERES-2-Cex06-11-56.

\section{References}

1. Gurtin, M.E.: The linear theory of elasticity. In: Truesdell, C. (ed.) Handbuch der Physik, vol. VIa/2. Springer, Berlin Heidelberg New York (1972)

2. Merodio, J., Ogden, R.W.: Instabilities and loss of ellipticity in fiber-reinforced compressible nonlinearly elastic solids under plane deformation. Int. J. Solids Struct. 40, 4707-4727 (2003)

3. Chiriţă, S., Ciarletta, M.: Spatial estimates for the constrained anisotropic elastic cylinder. J. Elast. 85, 189-213 (2006)

4. Simpson, H.C., Spector, S.J.: On copositive matrices and strong ellipticity for isotropic elastic materials. Arch. Ration. Mech. Anal. 84, 55-68 (1983)

5. Dacorogna, B.: Necessary and sufficient conditions for strong ellipticity of isotropic functions in any dimension. Dyn. Syst. Ser. B 1, 257-263 (2001)

Springer 
6. Payton, R.G.: Elastic Wave Propagation in Transversely Isotropic Media. Martinus Nijhoff, The Hague (1983)

7. Padovani, C.: Strong ellipticity of transversely isotropic elasticity tensors. Meccanica 37, 515-525 (2002)

8. Merodio, J., Ogden, R.W.: A note on strong ellipticity for transversely isotropic linearly elastic solids. Q. J. Mech. Appl. Math. 56, 589-591 (2003)

9. Chiriţă, S.: On the strong ellipticity condition for transversely isotropic linearly elastic solids. An. St. Univ. Iasi, Matematica, f.2, 52, 113-118 (2006)

10. Evans, K.E.: Auxetic polimers: a new range of materials. Endeavour, New Series, 15, 170-174 (1991)

11. Gibson, L.J., Ashby, M.F.: Cellular Solids-Structure and Properties, 2nd edn. Cambridge Press, UK (1997)

12. Scarpa, F., Tomlinson, G.: Theoretical characteristics of the vibration of sandwich plates with in-plane negative Poisson's ratio values. J. Sound Vib. 230, 45-67 (2000) 